LBL -32728

DE93 000580

\begin{abstract}
Development and Application of the
Quasi-potential Transformation
\end{abstract}

Bavanethan Pillay

(M.S. Thesis)

\author{
Department of Chemical Engineering \\ University of California \\ and \\ Materials Sciences Division \\ Lawrence Berkeley Laboratory \\ University of California \\ Berkeley, California 94720
}

August 1992

This work was supported in part by the Assistant Secretary for Conservation and Renewable Energy, Office of Transportation Technologies, Electric and Hybrid Propulsion Division of the U. S. Department of Energy under Contract No. DE-AC03-76SF00098. 
Abstract

The quasi-potential transformation, which is based on the Kirchhoff transformation, reduces the equations governing masstransfer in a steady-state, nonconvective electrolytic system into two independent parts. The geometry-specific part involves the solution of Laplace's equation subject to the relevant boundary conditions. The system-specific part involves the solution of a set of coupled first-order, nonlinear, ordinary differential equations.

We develop a theoretical basis for the quasi-potential transformation using potential theory. The major assumption on which the quasi-potential transformation is based is that the concentrations can be written as single-valued functions of the electrostatic potential. We discuss the consistency of this assumption. In so doing we see how the system-specific part of the calculation is developed. The boundary conditions used in this work are outlined, and the geometry-specific calculations for the disk and hemisphere electrodes are developed. We combine the system-specific calculations for the binary and acidic copper sulfate solutions with these geometryspecific calculations to obtain complete concentration profiles, potential distributions, and current density distributions for these systems. We also investigate the effect of migration on limiting currents. 
Abstract 1

Table of Contents ii

List of Symbols iii

Acknowledgements vi

1.0 Introduction 1

2.0 Theoretical Development 5

3.0 Boundary Conditions $\quad 26$

4.0 Geometry-Specific Calculations 29

5.0 Binary Copper Sulfate 37

6.0 Acidic Copper Sulfate 48

7.0 Effect of Migration on Limiting Currents 59

$\begin{array}{ll}\text { Summary } & 67\end{array}$

References $\quad 70$

Appendix A. Computer Programs 73 


\section{List of Symbols}

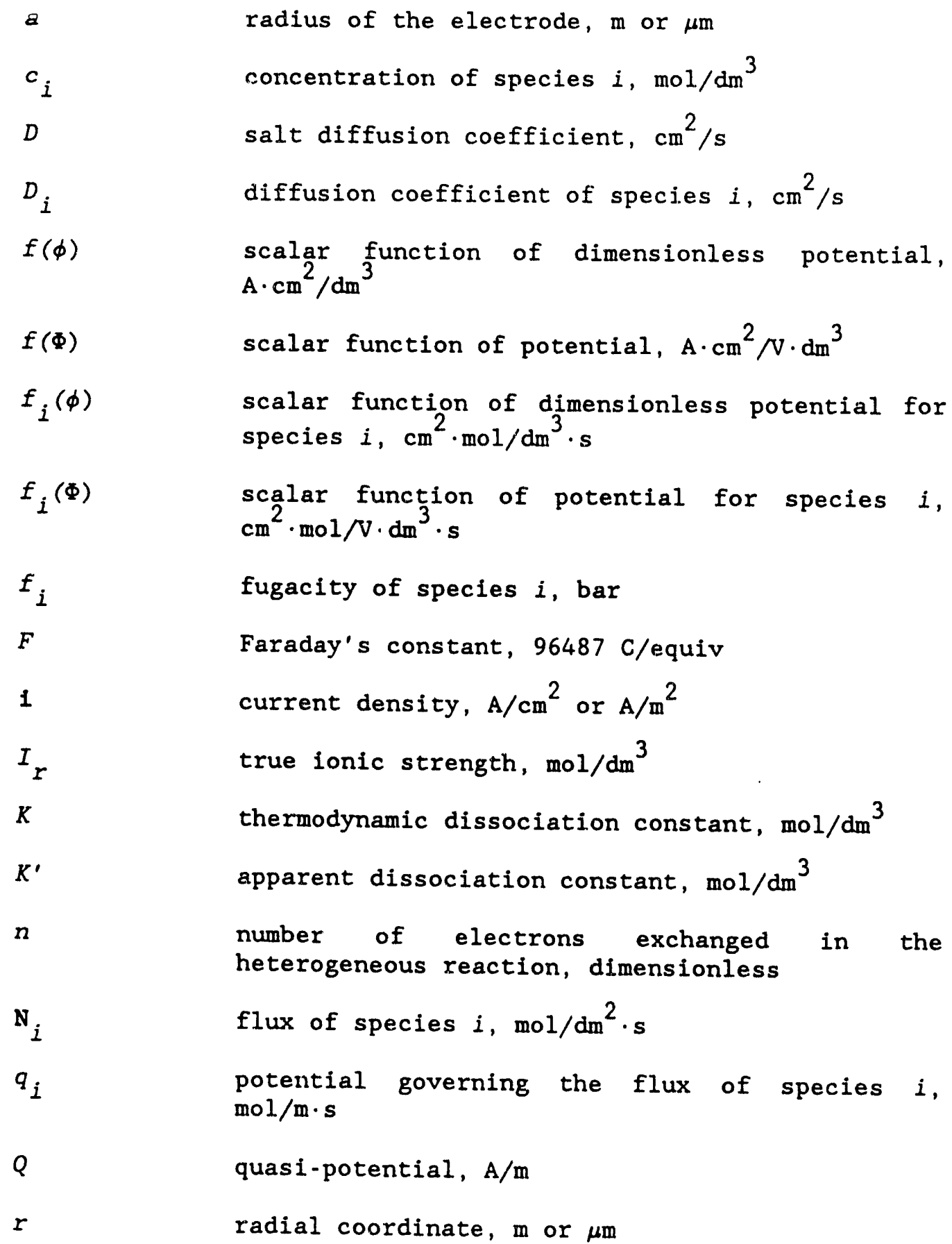




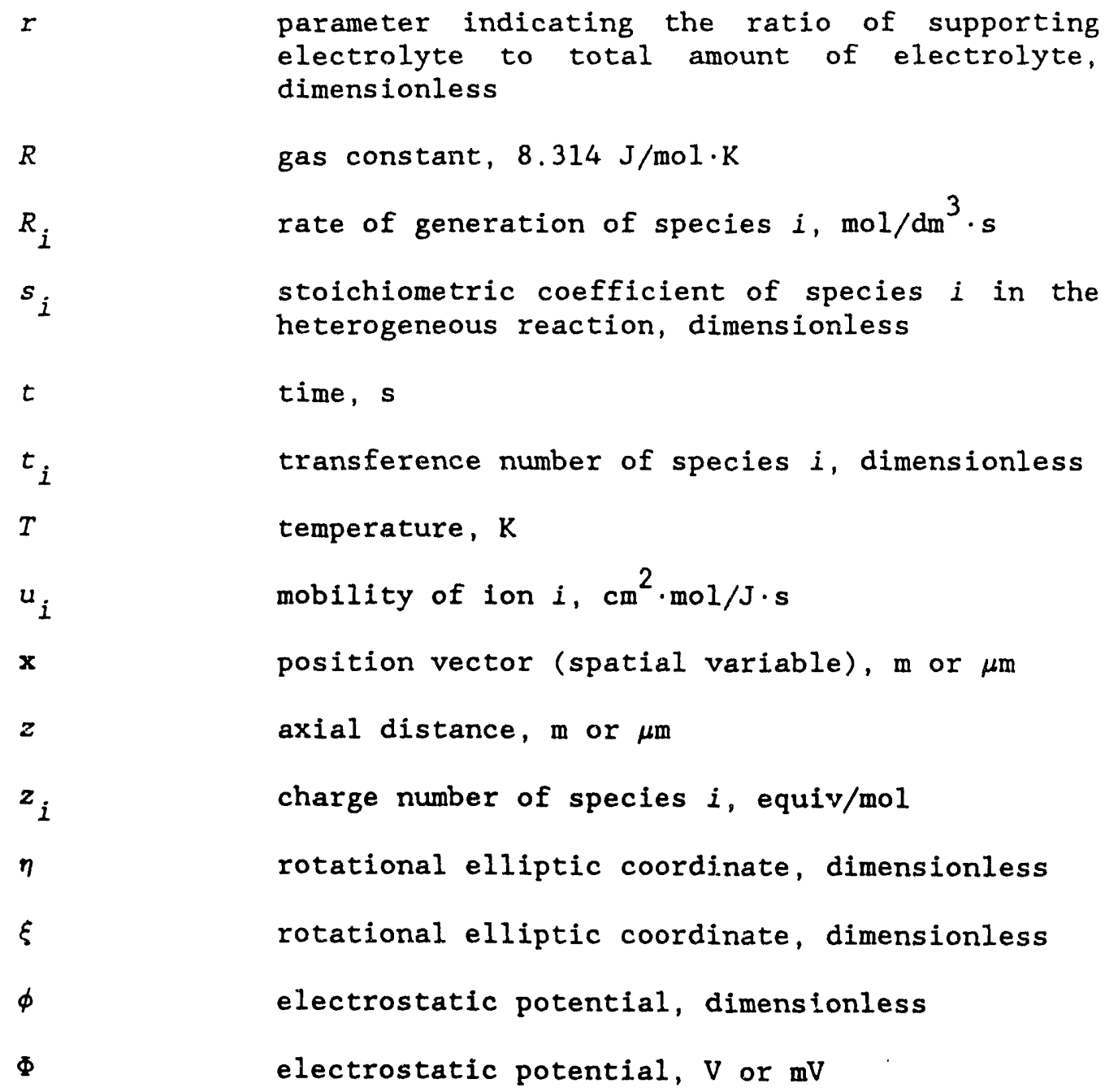

Subscripts

0

evaluated at electrode surface

avg average value

$D$

i evaluated with consideration of diffusion only 
$n$

$R$ normal component

principal reactant 


\section{Acknowledgements}

I would like to thank my research advisor, Professor John Newman, for his support in many different areas related to the completion of this thesis. I appreciate deeply the confidence he has shown in, and the patience he has shown to me. The members of my research group, especially Vince Battaglia and Tom Fuller who initiated me in the field of electrochemical engineering, have been a source of helpful criticism, inspiration, and entertainment.

Thanks are also due to the Educational Opportunities Council (South Africa), the Institute of International Education, the United Nations Educational and Training Programme for Southern Africa and the University of California at Berkeley for the financial support provided during the course of my Masters degree program.

This work was supported in part by the Assistant Secretary for Conservation and Renewable Energy, Office of Transportation Technologies, Electric and Hybrid Propulsion Division of the U. S. Department of Energy under Contract No. DE-AC03-76SF00098 
Determination of concentrations, potential, and current distribution is of major interest in the study of electrochemical systems. In their study of steady-state transport processes at stationary electrodes, Baker, Verbrugge, and Newman [1] introduced a powerful mathematical technique, based on the Kirchhoff transformation, which separates the set of coupled, nonlinear, second-order partial differential equations governing mass transfer into Laplace's equation and a set of coupled, initial-value, first-order, ordinary differential equations. They defined a new dependent variable which they called the quasi-potential.

It is the purpose of this work to develop a theoretical basis for this quasi-potential. We expand the previous work of these authors to show that potential theory applies rigorously in systems where the rate of mass transfer due to convection is negligible. We show that the gradient of the quasi-potential is a physically significant quantity, 2 ther than a mathematical invention, in that it can be defined as the same vector quantity as the current density. We also apply the quasi-potential transformation to two specific systems, allowing us to demonstrate the versatility of the technique. While our work here is based on dilute-solution theory, we feel that this technique might very well be applicable in situations where concentrated-solution theory is necessary.

There are a few limitations in the application of this technique. As stated previously, it can be applied only to systems in 
which convective contributions to the mass transport are negligible. Al1 systems must be at steady-state or quasi-steady-state. We have not, as yet, been able to use the quasi-potential transformation to solve a problem in which multiple independent heterogeneous reactions occur within the system. While the technique handles multiple homogeneous reactions easily, it is necessary that these reactions all be equilibrated.

With these limitations in mind, there are a few systems we identify here in which the quasi-potential transformation is applicable and useful.

In systems where both the electrode and the solution are stationary, there are no convective contributions to the mass transfer if natural convection and other effects, such as bulk convection due to large mass fluxes, are ignored. Any solutions derived for such electrode systems will be directly relevant to certain nonstationary microelectrode configurations, in which the velocities are moderate and the microelectrode is much smaller than the overall system comprising both the insulating plane and the electrode. We assume that all of the concentration variations take place in a region very close to the electrode surface, somewhat similar to a diffusion layer, and we refer to it as such. Since the microelectrode is on the order of micrometers and the overall system is on the order of centimeters, this diffusion layer is much smaller than the momentum boundary layer; hence the velocities in this diffusion layer are small relative to the velocities present in the bulk. In these 
microelectrode systems, the Péclet number in the diffusion layer is small enough that contributions to the mass transfer by convection can be ignored. This may be interpreted as a similar argument to the one used by Nernst in order to model mass transfer at the interface between a solid surface and a fluid. He postulated the existence of a stagnant diffusion layer adjacent to the surface. The argument used to reject this is based on the reasoning that for finite-sized electrodes the sizes of the diffusion and momentum boundary layers are not sufficiently different that the velocities become negligibly small within the diffusion layer (see as an example Levich [2]). Wightman and Wipf's review [3] provides an introduction to the field of microelectrodes.

Another system in which the quasi-potential is relevant is the corroding pit. Pitting corrosion has been observed to be particularly insidious in stagnant systems. Modeling of these pits has been of interest to electrochemical researchers for some time, but the resulting models have been complex or subject to a number of approximations. Sharland [4] has reviewed the modeling of pitting corrosion. Using the quasi-potential transformation, where applicable, one would be able to model pits simply, without resorting to many of the issumptions commonly used. Some common assumptions are the neglect of the effects of concentration gradients, the reduction of twodimensional problems to a single dimension, the omission of migration as a mechanism of mass transfer, and the use of constant physical properties. Many of the assumptions used are untenable. 
Other systems of possible interest are microscale etching processes (in the semiconductor industry), electropolishing, porous electrodes, cathodic protection of underground structures, and even pore-diffusion in nonelectrochemical systems.

In this work, we apply the quasi-potential transformation to two different stationary electrode geometries (these analyses would, of course, be relevant to some microelectrode systems as we11). The disk and hemisphere electrodes embedded in infinite insulating planes are both popular configurations for analytic and kinetic investigations. We solve these systems for the deposition of copper on a copper electrode from both binary and acidic aqueous $\mathrm{CuSO}_{4}$ solutions, subject to a few simple boundary conditions. We choose the binary $\mathrm{CuSO}_{4}$ solution because it is a well-studied, relatively simple system. The large amount of experimental physical data available for this system allows us to consider the effects of variable physical properties on transport rates in the system. We examine the acidic $\mathrm{CuSO}_{4}$ system since it is a system of some interest in plating and electrorefining. In treating the effect of the incomplete dissociation of bisulfate ions, we can show more fully how the quasipotential transformation handles homogeneous reactions. We do not consider the effects of variable physical properties in the acidic system as this would add a great deal of complexity to the problem, since there is no simple way to correlate the physical properties in terms of concentration. This is not a problem in a binary electrolyte system where there is just one independent concentration. 


\subsection{Theoretical Development}

The quasi-potential transformation divides the problem of mass transfer in nonconvective electrolytic (or nonelectrolytic) solutions into two parts.

The first part of the transformed problem depends only on the electrolytic species, the homogeneous reactions, and the heterogeneous reaction taking place in the system. It is independent of the geometry of the system. It involves manipulation of the material balance equations, the equation of electroneutrality, and those equilibrium relationships that are applicable. This part involves the solution of a set of coupled, initial-value, first-order, ordinary differential equations. From this part we determine the quasipotential and the species concentrations as functions of electrostatic potential, or the concentrations and electrostatic potential as functions of the quasi-potential. The results of these calculations may be used for all electrolytic systems of similar constitution and bulk composition, regardless of the geometry. In the work that follows we refer to this part of the calculation as the systemspecific part.

The second part of the problem involves solving Laplace's equation for the quasi-potential subject to the relevant boundary conditions, and hence accounts for the geometry. From this part we determine the quasi-potential as a function of the spatial variables. This part of the calculation is referred to as the geometry-specific 
part.

In this section we present the general equations governing mass transfer and current flow in the system. We outline the major assumption underlying the quasi-potential transformation. After using this assumption to show that the fluxes and current density are the gradients of potentials, we discuss its consistency.

The fundamental equations governing mass transfer in a continuum are given by Newman [5]:

Material balance:

$$
\frac{\partial c_{i}}{\partial t}=-\nabla \cdot \mathbf{N}_{i}+R_{i}
$$

Electroneutrality:

$$
\sum_{i} z_{i} c_{i}=0
$$

Current density:

$$
1-F \sum_{i} z_{i} N_{i}
$$

These equations apply regardless of whether dilute- or concentrated-solution theory is used to define $N_{i}$, the flux of species $i$. Newman also shows that

$$
\nabla \cdot 1=0
$$

which is a statement of the principle of conservation of charge. 
According to dilute-solution theory, the molar flux of species $i$ is given by

$$
\mathbf{N}_{i}=-z u_{i} F c_{i} \nabla \Phi-D_{i} \nabla c_{i}
$$

where the convective contribution to the flux has been neglected.

The critical assumption underlying the quasi-potential transformation is that $c_{i}(x)$ can be written as a single-valued function of $\Phi$; in other words, $c_{i}(x)$ is the composite function defined by

$$
c_{i}(x)=g_{i}(\Phi(x))
$$

We find it convenjent to refer to the function $g_{i}(\Phi)$ as $c_{i}(\Phi)$ since it is clear that $c_{i}(\Phi)$ has a different functional form from that of $c_{i}(x)$. We treat the physical properties in a similar manner.

We use this assumption that the concentration can be written as a single-valued function of the electrostatic potential on the basis that it gives a consistent solution to the governing equations. We cannot say unequivocally that this is the solution to the problem as we have not shown that it is a unique solution. A similar problem arises in the usage of many numerical techniques, where the solution that arises is consistent, but is very seldom proved to be unique. In that vein, we accept the solution generated by the quasi-potential transformation as our solution to the stated problem since it is a physically realistic solution.

We cannot use the assumption in systems where convection is important, since the presence of a velocity term (which is a function of the spatial variables) makes the resulting solution inconsistent 
with the governing equations. After we prove that the current (and the fluxes) is the gradient of a potential field, we shall show that the generated solution is consistent by showing that the concentrations that arise are returned as functions of the electrostatic potential only.

Applying the assumption that $c_{i}=c_{i}(\Phi)$, we get

$$
\mathbf{N}_{i}=f_{i}(\Phi) \nabla \Phi,
$$

where

$$
f_{i}(\Phi)=-z_{i} u_{i} F c_{i}-D_{i} \frac{d c_{i}}{d \Phi}
$$

To get this equation for $f_{i}(\Phi)$ we have made use of the chain-rule of differentiation. Many similar operations will be performed in later derivations.

The concentrations $c_{i}$ and their derivatives with respect to the electrostatic potential $\frac{d c_{i}}{d \Phi}$ and the transport properties-the transport coefficients $D_{i}$ and the ionic mobilities $u_{i}$-are all scalar. This implies that the function $f_{i}(\Phi)$ is a scalar function, and hence its derivative with respect to the electrostatic potential $\frac{d f_{i}}{d \Phi}$ is a scalar quantity.

Following the Kirchhoff transformation [6], as used by Baker, Verbrugge, and Newman [1], we define a new dependent variable as

$$
q_{i}=\int_{0}^{\Phi} f_{i}(\Phi) d \Phi .
$$

This allows us to write simply 


$$
\mathbf{N}_{i}=f_{i}(\Phi) \nabla \Phi=-\nabla q_{i},
$$

or using the equation for the current density (equation 3 ) and the property of the differential operator $\nabla$ that it operates linearly on sums

$$
1=f(\Phi) \nabla \Phi=-\nabla Q,
$$

where

$$
f(\Phi)=F \sum_{i} z_{i} f_{i}(\Phi)
$$

The negative sign has been included to meet with convention in the mass- (and heat-) transfer field that the flux density should be in the direction of the negative of the gradient of potential. Interestingly, this equation for the current density has a similar form to Ohm's law, with the analogous "conductivity" having an explicit dependence on the electrostatic potential.

The Kirchhoff transformation gives, very simply, our desired result. We have developed a more conventional proof, which is based on similar reasoning to the development of potential theory in fluid flow. This is a slightly more complicated proof, but we include it here since it raises a few important points about the quasi-potential transformation, which are not directly evident from using the Kirchhoff transformation.

If $\alpha$ is any scalar and $v$ is any vector, then

$$
\nabla \times \alpha v=\alpha(\nabla \times v)+(\nabla \alpha) \times v
$$

which is a general vector relationship. 
Now,

$$
\nabla \times 1-\nabla \times\left(\underset{i}{\sum_{i}} z_{i} \mathbf{N}_{i}\right)-F \sum_{i} z_{i}\left(\nabla \times \mathbf{N}_{i}\right)
$$

and

$$
\nabla \times \mathrm{N}_{i}=\nabla \times\left(f_{i} \nabla \Phi\right)-f_{i}(\nabla \times \nabla \Phi)+\left(\nabla f_{i}\right) \times \nabla \Phi .
$$

The curl of the gradient of a scalar is zero; therefore

$$
\nabla \times \mathbf{N}_{i}=\frac{d f_{i}}{d \Phi} \nabla \Phi \times \nabla \Phi
$$

The cross-product of two parallel vectors is zero; therefore

$$
\nabla \times \mathbf{N}_{i}=0
$$

and it follows that

$$
\nabla \times 1=0 .
$$

Batchelor [7] (p. 100) shows that for an irrotational, incompressible fluid the velocity is the gradient of a potential. Since the situation here is analogous, that proof can be used to show that the current density is the gradient of a potential. We reproduce that proof here as the use of Stokes's theorem has important consequences in the application of the quasi-potential theory.

Stokes's theorem states that

$$
\oint \mathbf{F} \cdot \mathrm{d} \mathbf{x}=\iint_{S} \mathrm{n} \cdot \nabla \times \mathbf{F} d S,
$$

where $F(x)$ is a vector function possessing continuous first partial derivatives and $\mathrm{n}$ is the unit vector normal to $S$, which is the portion of a regular surface bounded by the reducible, closed curve $C$. 
The term "reducible" is introduced by Batchelor (p. 92). He defines it as follows: a closed curve is reducible if it can be reduced to a point by a process of continuous deformation, without passing outside the region it is drawn in. Thus, if a reducible closed curve is drawn in some region, it is always possible to find an open surface which is bounded by the curve and which lies entirely within this region (for example, the surface being that traced out by the closed curve during its continuous reduction to a point). Gibbs [8] defines a region in which all closed curves are reducible as acyclic. All closed curves drawn within a siluply-connected region are reducible. The systems we consider in this work consist only of simply-connected regions. In multiply-connected regions some closed curves drawn in a single region are irreducible. Consider, as an example, a curve drawn around the surface of an infinitely long cylinder in an infinite fluid expanse. Stokes's theorem is inapplicable in such a situation, and we cannot assume that simple potential theory applies in such systems. It is probable that in multiplyconnected regions a number of potentials must be defined (this number being equal to the number of discontinuous regions). We believe this to be an important consideration in the application of the theory derived here.

Since $\nabla \times 1-0$ everywhere in the fluid, it follows from Stokes's theorem that

$$
\oint 1 \cdot d x=0 .
$$

If $O$ and $P$ are two different points in the fluid and $C_{1}$ and $C_{2}$ are 
two different paths joining $O$ to $P$, then, since $C_{1}$ and $C_{2}$ together constitute a closed, reducible curve passing through $O$ and $P$,

$$
\int_{C_{1}} 1 \cdot \mathrm{d} \mathbf{x}-\int_{C_{2}} 1 \cdot \mathrm{d} \mathbf{x}
$$

It follows that the line integral of 1 over any curve joining $O$ to $P$, lying entirely within the fluid, is the same for all members of a set of paths joining $O$ to $P$, and depends only on the position vectors of $O$ and $P, x_{O}$ and $x$ respectively. It is therefore possible to define a function $Q(\mathbf{x})$, which is a scalar function since the integral returns a scalar value, such that

$$
-Q(x)=-Q\left(x_{0}\right)+\int_{0}^{P} 1 \cdot d x,
$$

in which the integral is taken over any one of the paths in the set mentioned. Hence we find that

$$
I(x)=-\nabla Q(x),
$$

which defines the quasi-potential.

The second important consideration for our work stems from the fact that the choice of $x_{0}$ (and the choice of the value of $Q$ at this point) is arbitrary, since the difference between the values of $Q$ corresponding to two different choices of $x_{O}$ is independent of $\mathbf{x}$ (and the corresponding choice of $\left.Q\left(x_{0}\right)\right)$.

Consider starting points, $O$ and $O^{\prime}$, with position vectors, $x_{O}$ and $\mathbf{x}_{O}$ ', which are chosen arbitrarily (as are the values of $Q$ at these points). Then it follows that 
$Q^{\prime}(\mathbf{x})-Q\left(\mathbf{x}_{O}^{\prime}\right)-\int_{O^{\prime}}^{P} 1 \cdot \mathrm{d} \mathbf{x}=Q\left(\mathbf{x}_{O^{\prime}}\right)+Q\left(\mathbf{x}_{O}\right)-Q\left(\mathbf{x}_{O^{\prime}}\right)-\int_{O^{\prime}}^{O} 1 \cdot \mathrm{d} \mathbf{x}-\int_{O}^{P} 1 \cdot \mathrm{d} \mathbf{x}$, hence

$$
Q^{\prime}(x)-Q(x)-Q\left(x_{0}{ }^{\prime}\right)-Q\left(x_{0}\right)-\int_{O^{\prime}}^{0} 1 \cdot d x,
$$

where the prime on the $Q$ is used to indicate that $Q^{\prime}(x)$ is evaluated using the starting point $O^{\prime}$. Since the right side of this equation is independent of $x$, we see that

$$
\nabla Q^{\prime}(\mathbf{x})=\nabla Q(\mathbf{x})
$$

We can arbitrarily choose the value of the quasi-potential at any one point in such a way as to obtain amenable boundary conditions. This will only add a constant to the value of the quasi-potential. It will not affect its gradient which, as the negative of the current density, is of physical significance. We often choose to set the quasi-potential to zero infinitely far from the electrode surface.

Since the divergence of the current density is zero and the current density is the gradient of the quasi-potential,

$$
\nabla^{2} Q=0
$$

The quasi-potential obeys Laplace's equation in the fluid.

To relate the quasi-potential to the electrostatic potential, we see from the equation of current density (equation 3 ) and the derived equations 6,7 , and 9 that

$$
1-F \sum_{i} z_{i} N_{i}=f(\Phi) \nabla \Phi=-\nabla Q
$$

where 


$$
f(\Phi)=F \sum_{i} z_{i} f_{i}(\Phi)
$$

This is the same result that we obtain using the Kirchhoff transformation. It follows also that

$$
\mathbf{N}_{i}=f_{i}(\Phi) \nabla \Phi=-\nabla q_{i} .
$$

We may write

$$
\frac{d Q}{d \Phi}=-F \sum_{i} z_{i} f_{i}(\Phi)=-f(\Phi)
$$

which defines a first-order ordinary differential equation in $\Phi$ for $Q$.

In all of the work that follows, we assume that the stokesEinstein equation

$$
D_{i}=R T u_{i}
$$

holds. We write a nondimensional electrostatic potential as

$$
\phi=\frac{F \Phi}{R T}
$$

The equation for the flux becomes

$$
\mathbf{N}_{i}=-z_{i} D_{i} c_{i} \nabla \phi-D_{i} \nabla c_{i},
$$

or in terms of the electrostatic potential

$$
\mathbf{N}_{i}-f_{i}(\phi) \nabla \phi
$$

where

$$
f_{i}(\phi)=-D_{i}\left(z_{i} c_{i}+\frac{d c_{i}}{d \phi}\right)
$$

Also, 


$$
\frac{d Q}{d \phi}=-F \sum_{i} z_{i} f_{i}(\phi)=-f(\phi)
$$

We now show that the assumption we have used, that the concentration is a single-valued function of the electrostatic potential, results in a consistent solution by determining the concentrations that result from the system-specific calculation. This section is important also because the methods we develop here are used in all system-specific calculations. These methods are an essential part of the quasi-potential transformation.

Central to the system-specific calculation is a theorem presented by Gibbs [8] (page 39, Theorem 81):

If throughout a certain space (which need not be continuous and which may extend to infinity)

$$
\nabla \cdot \nabla u=0,
$$

and in all the bounding surfaces the normal component of $\nabla u$ vanishes, and at infinite distances within the space (if such there are) $r \frac{2 d u}{d r}=0$, where $r$ denotes the distance from a fixed origin, then throughout the space

$$
\nabla u=0,
$$

and in each continuous portion of the same

$$
u \text { - constant. }
$$

We have shown that each species flux may be written as the gradient of a potential. Since the gradient is a linear operator, it follows that any linear combination of fluxes may be written as the gradient of some single potential. If the species does not participate in a homogeneous reaction, or if the fluxes in the linear combination are combined in such a manner that the same linear combination 
of the species material balances results in the generation terms being eliminated from the resultant "combined" material balance, then the species flux, or the resultant flux formed by the linear combination, is solenoidal. For brevity, in the discussion that follows, we shall refer only to the flux of a single speciss, in the understanding that the conclusions drawn will be equally applicable to the resultant flux formed by the linear combination.

On all insulating surfaces the normal component of the species flux must be zero. If the electrode is not a source of this species, that is, the species does not participate in the heterogeneous reaction, then on the electrode surface the normal component of the species flux is also zero. This satisfies the condition in the theorem as regards [finite] bounding surfaces. The condition required at infinite distances is slightly more subtle. Consider that in any system, as one progresses farther and farther from the electrode, the electrode looks more and more like a point source. Choose a finite distance $R$, very far from the electrode but short of infinity. To an observer beyond $R$ the field generated by the electrode will look approximately similar to that generated by a hemisphere centered on the electrode, passing through $R$. Solving Laplace's equation $\nabla \cdot \nabla u$ for a spherical source one finds that $r^{2} \frac{d u}{d r}=$ constant. If the electrode is not a source, that is the normal component of $\nabla u$ is zero on the electrode, and if it is zero on all other boundaries at a finite distance from the electrode as well, then it also zero at the spherical surface, so that the spherical 
surface is not a source and one finds that $r^{2} \frac{d u}{d r}-0$. This approximation becomes exact as $R$ tends to infinity, and we see that the condition that $r^{2} \frac{d u}{d r}=0$ at infinity is satisfied if there is in fact no "source," that is, the normal component of $\nabla u$ is zero on all boundaries at a finite distance, and there are no external fields present in the system which may cause Laplace's equation for the potential (as defined above) to be inapplicable.

We have not shown conclusively that the existence of an external field precludes the applicability of the theorem of Gibbs; rather we have shown that the theorem holds in the absence of external fields. In all the work that follows, we assume that the only field present is due to the potential difference between the electrode and the counterelectrode at infinity and that there are no external fields present in the system - the condition that $r^{2} \frac{d u}{d r}=0$ at infinity holds, since $u$ will always be defined such that the normal component of $\nabla u$ is zero on all finite boundaries. Note that if the condition on the electrode (and naturally on the insulating plane) is that the normal component of $\nabla u$ is zero, and if $\nabla u$ represents a flux, then at a counterelectrode at finite distances the normal component of $\nabla u$ must be zero, to preserve the steady-state in the system.

Ionic species that do not participate in any of the homogeneous reactions (if such there are) or in the heterogeneous reaction we call stagnant, in that their fluxes are zero at all points in the system. For any species that does not participate in homogeneous reactions, the material balance equation reduces to 


$$
\nabla \cdot \mathbf{N}_{i}=0
$$

or writing this in terms of a potential

$$
\nabla \cdot \nabla q_{i}=0
$$

If this species does not participate in the heterogeneous reaction, then the normal component of its flux is zero at all finite surfaces, and if the counterelectrode is at infinity, the condition that $r^{2} \frac{d u}{d r}-0$ holds by the reasoning above. Therefore, whether the system is finite or infinite, the theorem of Gibbs holds, and it follows that

$$
\mathbf{N}_{i}=0,
$$

everywhere. Hence,

$$
f_{i}(\phi)--D_{i}\left(z_{i} c_{i}+\frac{d c_{i}}{d \phi}\right)=0 .
$$

For stagnant ions then

$$
c_{i}-c_{i}^{\infty} e^{-z \phi},
$$

where the integration constant has been evaluated using the condition that when $\phi=0, c_{i}-c_{i}^{\infty}$ (at infinity). We see that the concentration of any stagnant species satisfies a Boltzmann distribution.

If there are no homogeneous reactions, then the only nonstagnant ions are those that participate in the heterogeneous reaction. We choose arbitrarily one of the ions to be the principal reactant and relate the concentrations of the other nonstagnant species to the concentration of the principal reactant. Let the heterogeneous reaction be 


$$
s_{R} A_{R}+\sum_{j \neq R} s_{j} A_{j}=n e^{-}
$$

where the arbitrarily chosen principal reactant is designated by the subscript $R$, and the other ions that participate in the heterogeneous reaction are designated by the subscript $j$; we want to write all the $c_{j}$ as functions of $c_{R}$, and $c_{R}$ as a function of $\phi$ which will give us all the species concentrations as functions of the electrostatic potential. For each ion $j$ we may write a flux defined as

$$
\mathrm{N}_{j}-\frac{s_{j}}{s_{R}} \mathrm{~N}_{R} .
$$

The normal component of this flux must be zero at the electrode surface to satisfy the stoichiometry of the heterogeneous reaction. It must also be zero at all insulating surfaces, and, if the counterelectrode is a finite distance from the working electrode, the above stoichiometry must be true there as well, so as to preserve the steady-state in the system. The normal component of this flux is then zero at all finite surfaces. Since $N_{R}$ and $N_{j}$ are both individually gradients of a potential, it follows that a linear combination of these two fluxes must also be the gradient of some potential. The theorem of Gibbs then tells us that

$$
\mathrm{N}_{j}-\frac{s_{j}}{s_{R}} \mathrm{~N}_{R}=0
$$

everywhere. From this result and equation 12

$$
f_{j}(\phi)-\frac{s}{s_{R}} f_{R}(\phi)=0,
$$

and from equation 13 


$$
-D_{j}\left(z_{j} c_{j}+\frac{d c_{j}}{d \phi}\right)+\frac{s_{j}}{s_{R}} D_{R}\left(z_{R} c_{R}+\frac{d c_{R}}{d \phi}\right)=0 .
$$

Solving for $\frac{d c j}{d \phi}$, we have

$$
\frac{d c_{j}}{d \phi}=-z_{j} c_{j}+\frac{s_{j}}{s_{R}} \frac{D_{R}}{D_{j}}\left(z_{R} c_{R}+\frac{d c_{R}}{d \phi}\right) .
$$

Upon combining each of these equations with the Boltzmann distribution for any stagnant ions present in the system, with the equation of electroneutrality, and with a differentiated equation of electroneutrality

$$
\sum_{i} z_{i} \frac{d c_{i}}{d \phi}=0
$$

we obtain a set of coupled initial-value, first-order, ordinary differential equations which may be solved by simple numerical techniques, to obtain a set of species concentrations as single-valued functions of electrostatic potential, consistent with our original assumption. Equation 14, the differential equation for $Q$ as a function of $\phi$ may be added to this set and solved simultaneously, to obtain the quasi-potential. The initial values of concentration to be used to solve this set of equations are those at infinity, where the electrostatic and quasi-potentials are zero.

The arithmetic becomes much more involved when homogeneous reactions occur in the system; however, the method remains essentially the same. The material-balance equations are combined linearly to eliminate the generation terms. Those particular material-balance equacions eliminated in this procedure are replaced by the applicable equilibrium relationships. If any of the species present in the 
combined material balance participate in the heterogeneous reaction, then the same techniques as used above are applicable; however, the solenoidal flux vector is defined by the combined material balance of both the ion in question and that of the principal reactant. This can be involved, particularly if the principal reactant participates in the homogeneous reactions. If none of the ions present in the combined material balance participates in the heterogeneous reaction, then the solenoidal vector defined by the combined material balance is zero everywhere.

We have shown the consistency of using the assumption that the concentrations are single-valued functions of the electrostatic potential. We could also show, in exactly the same manner, that the electrostatic potential and species concentrations may be written as single-valued functions of the concentration of a single, arbitrarily chosen ionic species. This may be useful in two situations - in nonelectrochemical systems where the electrostatic potential is constant, or not defined, throughout the system and hence irrelevant to the mass transfer, and in systems of binary electrolytes where, at limiting-current conditions, the electrostatic potential is infinite at the disk surface.

The methods of determining the species concentrations as functions of the electrostatic potential, essential to the systemspecific part of the calculation, are written rather generally, and may appear vague. We hope that these ideas will be clear when they are applied to specific systems later in this work. The generality 
of these methods suggests that a general algorithm may be written to deal with the system-specific part oi the calculation, for any system consisting of an arbitrary number of ions and homogeneous reactions. As of yet such an algorithm has not been written; it is set aside as future work. We now derive general equations for a binary electrolyte system having one stagnant ion.

Binary Systems: There can be no homogeneous reaction taking place in a binary system, without the creation of another phase, since this would necessitate the presence of more than two species in solution. often, only one ion will participate in the heterogeneous reaction; therefore the other ion must be stagnant. We designate the stagnant ion to be species 2, and the fon that participates in the heterogeneous reaction we designate to be species 1 . The stagnant ion obeys a Boltzmann distribution

$$
c_{2}=c_{2}^{\infty} e^{-z} 2^{\phi}
$$

The condition of electroneutrality demands that

and hence

$$
z_{1} c_{1}--z_{2} c_{2}
$$

$$
c_{1}=c_{1}^{\infty} e^{-z} 2^{\phi}
$$

hence

$$
\phi=-\frac{1}{z_{2}} \ln \left(\frac{c_{1}}{c_{1}^{\infty}}\right) .
$$

Since we are using a species concentration as the independent 
variable, we write the flux as

$$
\mathrm{N}_{i}-h_{i}\left(c_{i}\right) \nabla c_{i}
$$

and from equation (11)

In this particular case

$$
h_{i}\left(c_{i}\right)=-D_{i}\left(z_{i} c_{i} \frac{d \phi}{d c_{i}}+1\right) .
$$

$$
1--\nabla Q-F \sum_{i} z_{i} \mathrm{~N}_{i}-F z_{1} h_{1}\left(c_{1}\right) \nabla c_{1}
$$

therefore

$$
\frac{d Q}{d c_{1}}=z_{1} F D_{1} \frac{\left(z_{2}-z_{1}\right)}{z_{2}} .
$$

From the definition of the transference number,

$$
t_{i}=\frac{z_{i}^{2} u_{i} c_{i}}{\sum_{j} z_{j}^{2} u_{j} c_{j}}
$$

and the definition of the diffusion coefficient of the salt in a binary electrolyte system

$$
D=\frac{z_{1} u_{1} D_{2}-z_{2} u_{2} D_{1}}{z_{1} u_{1}-z_{2} u_{2}},
$$

we

find an expression for the diffusion coefficient of the ionic species 1 , in terms of these tabulated properties

$\dagger$ This salt diffusion coefficient dates back to Nernst, and is defined $\because$ Newman [5]. It is an observed diffusion coefficient that is a compromise between the individual diffusion coefficients of the anion and the cation, and is the diffusion coefficient that is normally reported in the literature on experimental investigations of diffusion in binary electrolytes. 


$$
D_{1}-\frac{z_{2}}{\left(z_{2}-z_{1}\right)} \frac{D}{\left(1-t_{1}\right)} \text {. }
$$

Substituting we obtain

$$
\frac{d Q}{d c_{1}}-z_{1} F \frac{D}{\left(1-t_{1}\right)}
$$

If the transport properties are considered to be constant, then we can integrate this equation analytically to obtain

$$
Q=z_{1} F \frac{D}{\left(1-t_{1}\right)}\left(c_{1}-c_{1}^{\infty}\right) \text {, }
$$

after applying the boundary condition that $Q$ is 0 when $\phi$ is 0 (at infinity). If we wish to account for the variations in the transport properties, then we need to integrate this differential equation numerically, using the boundary condition given, and substituting the tabulated values for the transport properties as functions of concentration. Since the equation governing the concentration of an ionic species in a binary electrolyte derived from dilute-solution theory is very similar to that derived from concentrated-solution theory (see Newman [5], page 269), the solution found here is similar to that that would be found using concentrated-solution theory.

We close this section by checking that the number of equations used matches the number of unknowns; namely, that our problem is properly specified. We start out with $n+2$ equations, where $n$ is the number of species present in the system, excluding the solvent. These $n+2$ equations are the $n$ equations of material balance (equations 1), the equation of electroneutrality (equation 2), and the vector equation defining the vector current density (equation 3). 
The number of unknowns is $n+2$ - the $n$ concentrations, the electrostatic potential, and the vector current density. To this set we add an unknown - the quasi-potential. In so doing, we add one more equation since we define the quasi-potential by setting its gradient to be the negative of the vector current density (equation 9). Thus, we have $n+3$ equations in $n+3$ unknowns.

We used all the equations above to show that the divergence and the curl of the current density are zero, and that, therefore, the quasi-potential satisfies Laplace's equation. We include Laplace's equation for the quasi-potential in our set to bring the number of equations to $n+4$, of which only $n+3$ are independent. As we see in the system-specific part of the calculation, we do not use the material balance for the principal reactant directly, hence we eliminate this equation, in favor of Laplace's equation which it was used to derive. The vector equations defining the vector current density in terms of the fluxes and the quasi-potential are combined to find the quasi-potential as a function of electrostatic potential. Since only 2 of the resulting 3 equations are independent, we can eliminate one of the vector equations defining the vector current density.

We thus have $n+3$ equations ( $n-1$ material balances, electroneutrality, Laplace's equation for the quasi-potential, a vector equation defining the vector current density, and an equation relating the quasi-potential to the electrostatic potential) in $n+3$ unknowns (n species concentrations, the quasi-potential, the electrostatic potential, and the vector current density). Laplace's equation (when 
solved with the relevant boundary conditions, some of which may come from the system-specific part of the calculation) constitutes the geometry-specific part of the problen. All the other equations constitute the system-specific part of the calculation.

\subsection{Boundary Conditions}

In the problems pursued here, we ignore any effect of the counterelectrode by assuming it to be a hemisphere located infinitely far from the working electrode. This is not necessary to the treatment, but is convenient as it simplifies the situation considerably. It is normally reasonable to ignore the presence of the counterelectrode, as most of the variations of concentration and potential take place in regions close to the working electrode. We set the electrostatic potential infinitely far from the electrode surface to zero. At infinity, the concentration of the individual species are set to their bulk values, and we set the value of the quasi-potential to be zero.

The rest of the necessary boundary conditions allow us to solve Laplace's equation for the quasi-potential. On all insulating surfaces, the current normal to the surface is zero. Since $1--\nabla Q$,

$$
i_{n}=-\frac{\partial Q}{\partial n}=0,
$$

where $\frac{\partial Q}{\partial n}$ is the component of the gradient normal to the surface.

The boundary conditions at the electrode surface depend on the physical situation in the system. We outline here the boundary 
conditions used in this work, and the values that the quasi-potential or its derivative assume at the surface for each condition. For a more comprehensive treatment of these types of boundary conditions see Newman [5]. West and Newman [9] have written a helpful review on solving for current distributions governed by Laplace's equation. They discuss the boundary conditions as well as means of solving Laplace's equation subject to these boundary conditions. This paper (and the references it provides) is particularly valuable to this work, as many of the methods outlined there are directly applicable.

1) Limiting-Current Distribution: The limiting-current distribution occurs when the concentration of the principal reactant participating in the electrode reaction becomes zero adjacent to the electrode. The current is limited by the transport of reactant to the surface, hence the name, mass-transfer-limited current or simply, limiting-current. It is significant in that it is the maximum possible current that can be transferred in the system, if no other heterogeneous reactions take place. Since lines of constant electrostatic potential are also lines of constant concentration and constant quasi-potential, the quasi-potential becomes constant at the surface. This boundary condition, stated mathematically, is

$$
Q_{0}-\text { constant, }
$$

where $Q_{0}$ is the value of the quasi-potential at the electrode surface (just outside the electric double-layer). The indicated constant is to be determined from the first part of the solution, namely the system-specific part which generates the quasi-potential and 
concentrations as functions of the electrostatic potential. This constant is independent of the geometry of the system.

2) Uniform-Potential Distribution: When the surface overpotential is negligible, the potential adjacent to an electrode may be taken as uniform. Use of this boundary condition generates the maximum variation in current density across the surface of the electrode. The problem generated by this boundary condition, when t're quasi-potential transformation is used, is virtually identical to that when the current distribution is mass-transfer limited since an equi-electrostatic potential surface is also an equi-quasi-potential surface. Again, we find that

$$
Q_{0}=\text { constant, }
$$

where the constant is obtained as in the limiting-current case. The limiting-current distribution case might be thought of as a specific case of the more general uniform-potential distribution case.

3) Uniform-Current-Density Distribution: The opposite extreme of the constant-potential distribution case is the case when there is a uniform-current-density distribution across the surface of the disk. This case corresponds, usually, to very slow electrode kinetics, and when there is a constant concentration in the solution it can be shown that this condition is met for very small exchange-current densities. While we have not shown that this condition is met generally for systems exhibiting concentration gradients, we investigate it nonetheless, since it is of some importance. This situation occurs obviously when the electrode surface is uniformly accessible to mass 
transfer (consider a hemisphere embedded in an infinite insulating plane). The situation of a uniform-current-density boundary condition corresponds to the situation where the variation of electrostatic potential across the surface of the electrode is at a maximum. We state this mathematically as

$$
\frac{\partial Q}{\partial n}=\text { constant, }
$$

where the constant is the negative of the value of the current density at the electrode surface (usually specified).

\subsection{Geometry-Specific Calculations}

In this section we discuss the solutions of Laplace's equation subject to the boundary conditions listed in section 3.0 . The solutions discussed here are for a disk electrode and a hemisphere electrode, both embedded in infinite insulating planes. For both systems the counterelectrode is considered to be a hemisphere located infinitely far from the electrode surface. As discussed briefly in section 3.0 , the boundary conditions on the electrode for the limitingcurrent cistribution and constant-potential distribution are very similar. In fact, the limiting-current distribution condition may be considered a special case of the constant-potential distribution condition. It follows that the same method of solution of Laplace's equation is applicable to both cases. Since all solutions for the hemisphere will satisfy the condition of uniform-current-density distribution due to its spherical symmetry, no special solution is 
required for the boundary condition of uniform-current-density distribution. It is evident that we have only three different methods of solution of Laplace's equation to consider - the disk with uniform-potential distribution, the disk with uniform-current-density distribution, and the hemisphere at uniform-potential distribution.

One of the important features of the quasi-potential transformation is highlighted here. We see that the methods of solving Laplace's equation are relevant to all electrode systems of the same geometry and boundary conditions, regardless of the specific electrolyte and solvent that are used in the system. Later in this work, we will use similar solutions of Laplace's equation to complete the concentration and potential distributions for both the acidic and binary copper sulfate systems.

The other important feature we would like to point out is that solutions of Laplace's equation with electrochemical-type boundary conditions, such as those listed in section 3.0 and more complex polarization conditions (for example, the Butler-Volmer type boundary condition), have been extensively studied for decades. If one ignores the concentration gradients in a system, then the electrostatic potential in that system is governed by Laplace's equation. These solutions are not rigorously correct, but there exist many systems (example, well-stirred systems) where the actual behavior is closely approximated by these solutions, and for exceedingly complex systems, these solutions give an indication of the system behavior. While recognizing the shortcomings of their solutions, many authors 
have solved Laplace's equation for the electrostatic potential, because the rigorous solution was too difficult or impossible to obtain. These solutions-primary-current distributions, secondarycurrent distributions, and certain limiting-current distributionsare directly usable together with the quasi-potential transformation to obtain rigorous solutions for nonconvective systems. In this work, the methods of solution we use for the disk come directly from such previous work. It is beyond the scope of this thesis to provide a complete review of this previous work, because there are many hundreds of papers detailing such work in the literature. We have found that the review of West and Newman [9], which we have previously cited, to be of use. Extremely useful was the work of Newman [10], which is a review of mathematical methods in electrochemical engineering.

The Disk Electrode: For the disk we discuss the solutions of Laplace's equation for the constant-potential distribution and for the uniform-current-density distribution.

Constant-Potential Distribution: Newman [11] has solved Laplace's equation $\nabla^{2} \Phi=0$ for the disk electrode in an infinite insulating plane with the counterelectrode being a hemisphere infinitely far from the electrode surface, using rotational elliptic coordinates

$$
z=a \xi \eta
$$




$$
r=a \sqrt{\left(1+\xi^{2}\right)\left(1-\eta^{2}\right)}
$$

where $a$ is the radius of the disk. In this coordinate system, for an axially symmetric system, Laplace's equation takes the form

$$
\frac{\partial}{\partial \xi}\left[\left(1+\xi^{2}\right) \frac{\partial \Phi}{\partial \xi}\right]+\frac{\partial}{\partial \eta}\left[\left(1-\eta^{2}\right) \frac{\partial \Phi}{\partial \eta}\right]=0,
$$

and the boundary conditions used by Newman take the form

$$
\begin{aligned}
& \Phi=\Phi_{0} \text { at } \xi=0 \text { (on the disk electrode) } \\
& \frac{\partial \Phi}{\partial \eta}=0 \text { at } \eta=0 \text { (on the insulating annulus) } \\
& \Phi=0 \text { at } \xi=\infty \text { (far from the disk) }
\end{aligned}
$$

$\Phi$ well behaved at $\eta-1$ (on the axis of the disk).

The method of separation of variables results in solutions in terms of Legendre functions. Since the equation and the boundary conditions are exactly the same for our system, the same solution applies

$$
Q / Q_{0}=1-\left(\frac{2}{\pi}\right) \tan ^{-1} \xi
$$

Newman [10] discusses the entire solution in more detail.

We need to determine the value of $Q_{0}$, the value of the quasipotential at the disk surface. To do this we use the system-specific calculations. If the disk is at limiting-current conditions, the value of $Q_{0}$ is found by evaluating $Q$ when the concentration of the principal reactant is set to zero. For a uniform-potentialdistribution condition, the value of the electrostatic potential at the disk surface is given; $Q_{0}$ is the value that $Q$ assumes when the electrostatic potential assumes this value. 
of interest here is the determination of the radial current distribution on the disk surface. We find that

$$
i_{n}=\frac{2 Q_{0} / \pi}{\sqrt{a^{2}-r^{2}}}
$$

Integrating this equation over the disk surface and dividing by the area, we obtein for the average current

$$
i_{\text {avg }}=\frac{4 Q_{0}}{\pi a}
$$

It could also be shown that lines of constant $\eta$ are what are termed current lines, in that they are similar to streamlines in fluid flow - there is no flow of current across them. Furthermore, the values of $\eta$ corresponding to a particular current line indicates the fraction of total current flowing through the electrolyte between the current line and the plane $z=0$.

Constant Current Density Distribution: Nanis and Kesselman [12] have obtained solutions to Laplace's equation for the same disk geometry for a few different boundary conditions. A similar solution is discussed in detail by Wylie and Barrett [13] (example 7, page 620), and our solution follows theirs closely.

For an axially symmetric system, Laplace's equation in cylindrical coordinates is

$$
\frac{\partial^{2} Q}{\partial r^{2}}+\frac{1}{r} \frac{\partial Q}{\partial r}+\frac{\partial^{2} Q}{\partial z^{2}}=0
$$


The boundary conditions in cylindrical coordinates have the form

$$
\begin{aligned}
& \frac{\partial Q}{\partial z}=-i_{n} \text { for all } r<a, z-0 \text { (on the disk electrode) } \\
& \frac{\partial Q}{\partial z}=0 \text { for all } r>a, z-0 \text { (on the insulating annulus) } \\
& Q \rightarrow 0 \text { as } r^{2}+z^{2} \rightarrow \infty \text { (far from the disk) } \\
& Q \text { well behaved at } r=0, \text { all } z \geq 0 \text { (on the axis of the disk). } \\
& \text { After separating and applying the two radial boundary condi- }
\end{aligned}
$$
tions, we obtain

$$
Q_{\lambda}(r, z)=A(\lambda) J_{0}(\lambda r) e^{-\lambda z},
$$

where $A$ is a constant resulting from the integration. Integrating over all possible values of $\lambda$ to obtain the most general form of the solution, in order to satisfy the boundary condition on the electrode,

$$
Q(r, z)-\int_{0}^{\infty} A(\lambda) J_{0}(\lambda r) e^{-\lambda z} d \lambda .
$$

We want to satisfy the boundary condition

$$
\frac{\partial Q}{\partial z}(r, 0)-F(r)=-\int_{0}^{\infty} A(\lambda) \lambda J_{0}(\lambda r) d \lambda,
$$

where

$$
F(r)= \begin{cases}-i n & \text { if } r<a \\ 0 & \text { if } r>a\end{cases}
$$

The integral above may be recognized as one member of the Hankel transform pair for Bessel functions of order $\nu=0$, therefore 


$$
A(\lambda)=-\int_{0}^{\infty} r F(r) J_{0}(\lambda r) d r
$$

which reduces to

$$
A(\lambda)=\frac{a i_{n}}{\lambda} J_{1}(a \lambda) .
$$

The complete solution for the quasi-potential is then

$$
Q(r, z)=a i_{n} \int_{0}^{\infty} J_{0}(r \lambda) \frac{J_{1}(a \lambda)}{\lambda} e^{-\lambda z} d \lambda .
$$

The Weber-Schafheitlin formula (see Abramowitz and Stegun [14]) allows one to find the potential distribution on the disk surface and on the insulating plane (see Nanis and Kesselman). These results are

$$
Q(r, 0)=\left\{\begin{array}{ll}
\frac{2 a i n}{\pi} E\left[\frac{r^{2}}{a^{2}}\right) & , 0<r<a \\
\frac{2 r i n}{\pi}\left[K\left(\frac{a^{2}}{r^{2}}\right)-\left(1-\frac{a^{2}}{r^{2}}\right) E\left(\frac{a^{2}}{r^{2}}\right)\right] & , a<r<\infty
\end{array},\right.
$$

where $\mathrm{K}(\boldsymbol{m})$ is the complete elliptic integral of the first kind and $E(m)$ is the complete elliptic integral of the second kind as defined by Abromowitz and Stegun

$$
\mathbf{R}(m)-\int_{0}^{1}\left[\left(1-t^{2}\right)\left(1-m t^{2}\right)\right]^{-\frac{2}{2}} d t
$$

and

$$
\mathbf{E}(m)-\int_{0}^{1}\left(1-t^{2}\right)^{-\frac{1}{2}}\left(1-m t^{2}\right)^{\frac{1}{2}} d t .
$$

To obtain the concentrations, potential, and current distributions in the entire region of electrolyte, the integral needs to be evaluated in the form given. We expect that the integral is 
convergent since each of the terms within the integrand tends to 0 as $\lambda$ tends to $\infty$. The integral may be evaluated as $Q(\infty)$ when $Q(\lambda)$ is the solution of

$$
\frac{d Q}{d \lambda}=f(\lambda)=a i_{n} J_{0}(r \lambda) \frac{J_{1}(a \lambda)}{\lambda} e^{-\lambda z},
$$

subject to the initial condition

$$
Q=0 \text { at } \lambda=0 \text {. }
$$

This may be solved relatively simply, using the Runge-Kutta method. We find

$$
\lim _{\lambda \rightarrow 0} f(\lambda)=\frac{a^{2}{ }^{n} n}{2}
$$

by using l'Hopital's rule. This value is necessary when the RungeKutta technique is used.

The Hemisphere Electrode: The solution for the hemisphere electrode is simple. Laplace's equation in spherical coordinates, where the system is spherically symmetric, assumes the form

$$
\frac{1}{r^{2}} \frac{d}{d r}\left(r^{2} \frac{d Q}{d r}\right)=0
$$

The boundary conditions are

$$
\begin{aligned}
& Q=Q_{0} \text { at } r=a \text { (on the electrode surface) } \\
& Q \rightarrow 0 \text { as } r \rightarrow \infty \text { (far from the electrode), }
\end{aligned}
$$

where $a$ is the radius of the hemisphere. The solution is

$$
\frac{Q}{Q_{0}}=\frac{a}{r}
$$


and the current density on the electrode surface is

$$
i_{n}=\frac{Q_{0}}{a},
$$

which is also the average current density.

\subsection{Binary Copper Sulfate}

The mechanism of the copper deposition reaction is given by Mattsson and Bockris [15] and has been confirmed by numerous authors including Bertocei [16] and Brown and Thirsk [17]

$$
\begin{aligned}
& \mathrm{Cu}^{2+}+\mathrm{e}^{-} \rightarrow \mathrm{Cu}^{+} \text {(slow) } \\
& \mathrm{Cu}^{+}+\mathrm{e}^{-} \rightarrow \mathrm{Cu} \text { (fast). }
\end{aligned}
$$

All of these authors investigated the mechanism in dilute sulfuric acid solutions. We are unaware of a study of the deposition reaction in nonacidic solutions, but since there is no obvious reason to suspect that there should be any difference in the mechanism in the absence of acid, we accept this as being the mechanism of the deposition reaction in the binary aqueous copper sulfate system, as well as in the acidic aqueous copper sulfate system.

As previously indicated, we are unable to use the quasipotential transformation to solve a system which has two independent heterogeneous reactions taking place at the same time. We assume therefore, that the reaction of the cuprous ions to form metallic copper occurs rapidly, and that there is no diffusion of cuprous ions away from the electrode surface. We are assuming that the cuprous 
ions do not exist as an independent ionic species in the solution. Using this assumption, we write the deposition reaction as

$$
\mathrm{Cu}^{2+}+2 \mathrm{e}^{-} \rightarrow \mathrm{Cu}
$$

Since the second reaction in the sequence has been measured as being much faster than the first reaction, it is a fairly good assumption to write the deposition reaction in this form, and we shall use this form in the two systems we study here.

The first system we discuss is the binary copper sulfate solution, with the copper depositing on a copper electrode. We assume that the copper sulfate salt is completely dissociated into cupric and sulfate ions; there are no cuprous ions present in the solution. The equations we derived for binary electrolytes in section 2.0 are directly applicable here. From equation 19

$$
\frac{d Q}{d c_{1}}-2 F \frac{D}{\left(1-t_{1}\right)}
$$

where we have adopted the convention of that section by designating the cupric ions as species 1 and the sulfate ions as species 2 .

If we assume that the transport properties do not vary over the range of concentrations from that in the bulk to that adjacent to the surface, then equation 20 applies

$$
Q=2 F \frac{D}{\left(1-t_{1}\right)}\left(c_{1}-c_{1}^{\infty}\right)
$$

otherwise we need to insert correlations for the transport properties as functions of concentration into the differential equation before integrating. 
There is a large amount of data on the diffusion coefficient of the copper sulfate salt and the transference number of the cupric ion available in the literature. The paper by Miller et al. [18] provides a critical review of the diffusion-coefficient data then available. Since there is not a large amount of data that have been reported since then, and the data that have been reported since are not necessarily of greater accuracy, we choose to use the values of the diffusion coefficients reported in that paper. They used cubics and quadratics in the square root of concentration to fit the data over 3 separate data ranges. These correlations are

$$
\begin{array}{r}
10^{5} D=0.858-4.74668 c^{1 / 2}+54.1829 c-351.636 c^{3 / 2} \\
+1113.06 c^{2}-1348.34 c^{5 / 2} \quad(0 \leq c \leq 0.007), \\
10^{5} D=0.76408-1.16200 c^{1 / 2}+2.59446 c-2.90168 c^{3 / 2} \\
(0.007<c \leq 0.07),
\end{array}
$$

and

$$
10^{5} D=0.70490-0.50229 c^{1 / 2}+0.17844 c \quad(0.07<c<1.28),
$$

where $D$ is in $\mathrm{cm}^{2} / \mathrm{s}$ and $c$ is in mol. $\mathrm{dm}^{-3}$. In all of the work done here we have set the maximum bulk concentration of $\mathrm{CuSO}_{4}$ to be 1.40 mol. $\mathrm{dm}^{-3}$, which allows us to observe the maximum variations in concentration and potentials, subject to the concentration of $\mathrm{CuSO}_{4}$ staying below the limit of solubility $\left(1.4083 \mathrm{~mol} . \mathrm{dm}^{-3}\right)$. We feel confident in using the diffusion-coefficient correlation above the indicated range of applicability because Miller et al. indicate that 
the estimated error in the values of the diffusion coefficient obtained from these correlations above a $\mathrm{CuSO}_{4}$ concentration of 1.28 mol. $\mathrm{dm}^{-3}$ is less than 0.38 ; a value we have confirmed.

The transference-number data are taken from Pikal and Miller [19] and are correlated as a quadratic in the square root of concentration

$$
t_{1}=0.4069-0.1557 c^{\frac{1}{2}}+0.02551 c
$$

where, again, $c$ is in mol. $\mathrm{dm}^{-3}$.

The values of these properties, when averaged between $\mathrm{CuSO}_{4}$ concentrations of zero and $1.40 \mathrm{~mol} \cdot \mathrm{dm}^{-3}$ (the value of $\mathrm{CuSO}_{4}$ concentration in the bulk) are

$$
D_{\text {avg }}=0.436 \times 10^{-5} \mathrm{~cm}^{2} / \mathrm{s}
$$

and

$$
t_{1, \text { avg }}=0.302 \text {. }
$$

It is believed that it is more realistic to use these average values in the constant-physical-properties model, rather than those at infinite dilution. The quasi-potential evaluated with constant physical properties is given by

$$
Q=0.12054 c_{1}-0.16876(\mathrm{~A} / \mathrm{m}) \text {, }
$$

where $c_{1}$ is in mol. $\mathrm{dm}^{-3}$. When the variation of the physical properties is accounted for, the equation must be solved numerically. We use a fourth-order Runge-Kutta algorithm to evaluate the quasipotential. The pertinent computer programs are listed in Appendix A. 
Figure 1 shows the concentration and electrostatic potential as functions of the quasi-potential for both the variable- and constant-physical-property cases. For the limiting-current and uniform-potential distributions, we are interested in the maximum current that can be passed. Figure 2 shows the variation of the value of the quasi-potential on the disk surface with changes in the bulk concentration of $\mathrm{CuSO}_{4}$. The value of the average current density is directly related to the value of the quasi-potential on the surface by equations 23 and 27 . Note that the value of the average current density depends only on the system-specific part of the calculation through $Q_{0}$ (independent of the geometry), and depends on the geometry through the coefficient of $Q_{0}$ in equations 23 and 27.

The results of the system-specific part of the calculation shown in figure 1 are combined with the results of the geometry-specific calculations - the solutions of Laplace's equation for the disk and the hemisphere at constant-potential distribution, equations 21 and 26 - to give the concentration and electrostatic potential as functions of the spatial variables. These results are displayed in figure 3 and figure 4. We have used these results to generate the values of the concentration and electrostatic potential as functions of the cylindrical coordinates $r$ and $z$, which has enabled us to generate the two-dimensional pictures of the concentration and current distributions over the disk and the hemisphere, siown in figures 5 and 6 . The values given on these figures are the actual values of concentration generated by the solution of the equations. 


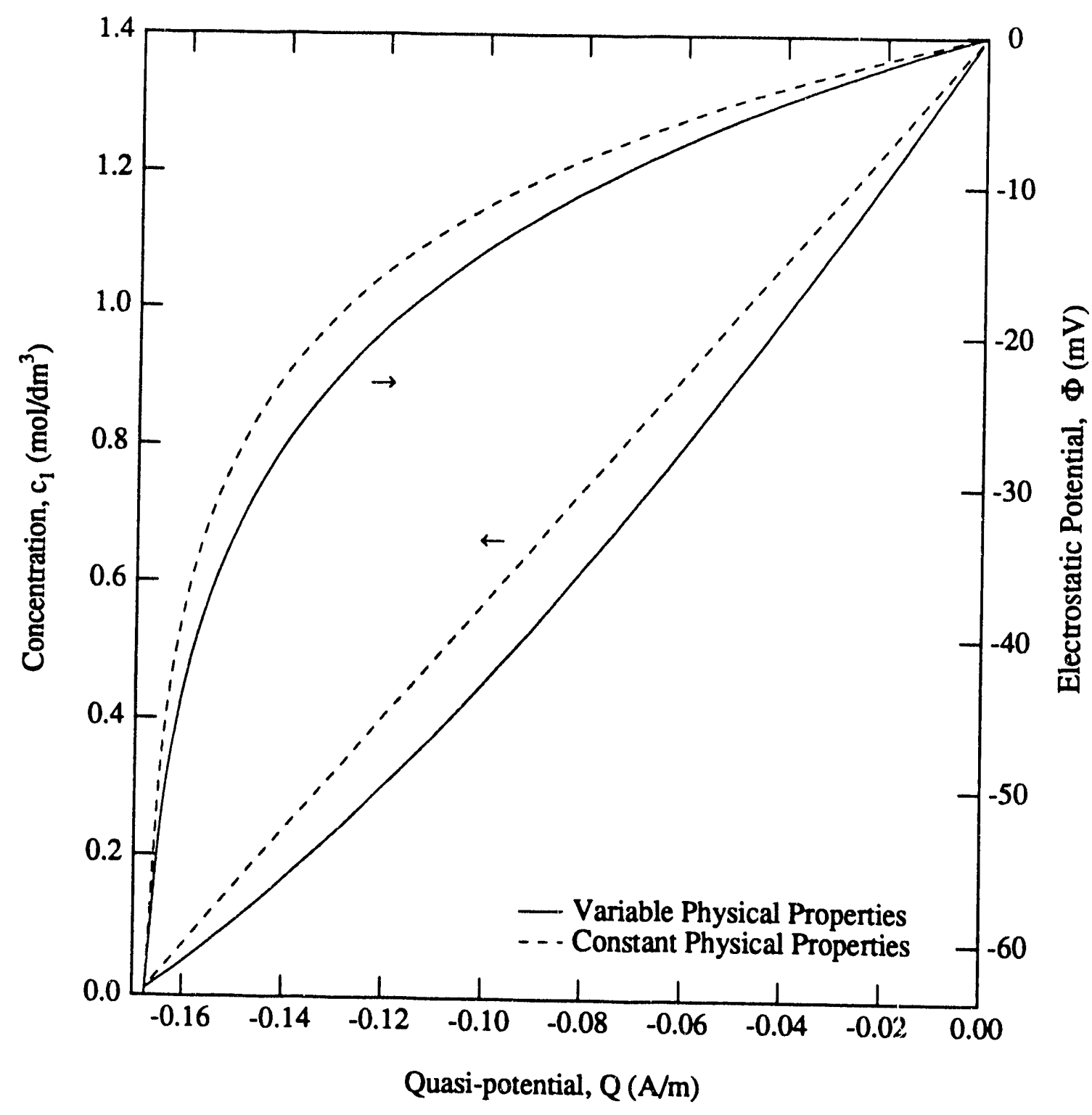

Figure 1. Electrostatic potential and concentration as functions of the quasi-potential in binary $\mathrm{CuSO}_{4}$ solution. The bulk concentration of $\mathrm{CuSO}_{4}$ is $1.40 \mathrm{~mol} / \mathrm{dm}^{3}$. 


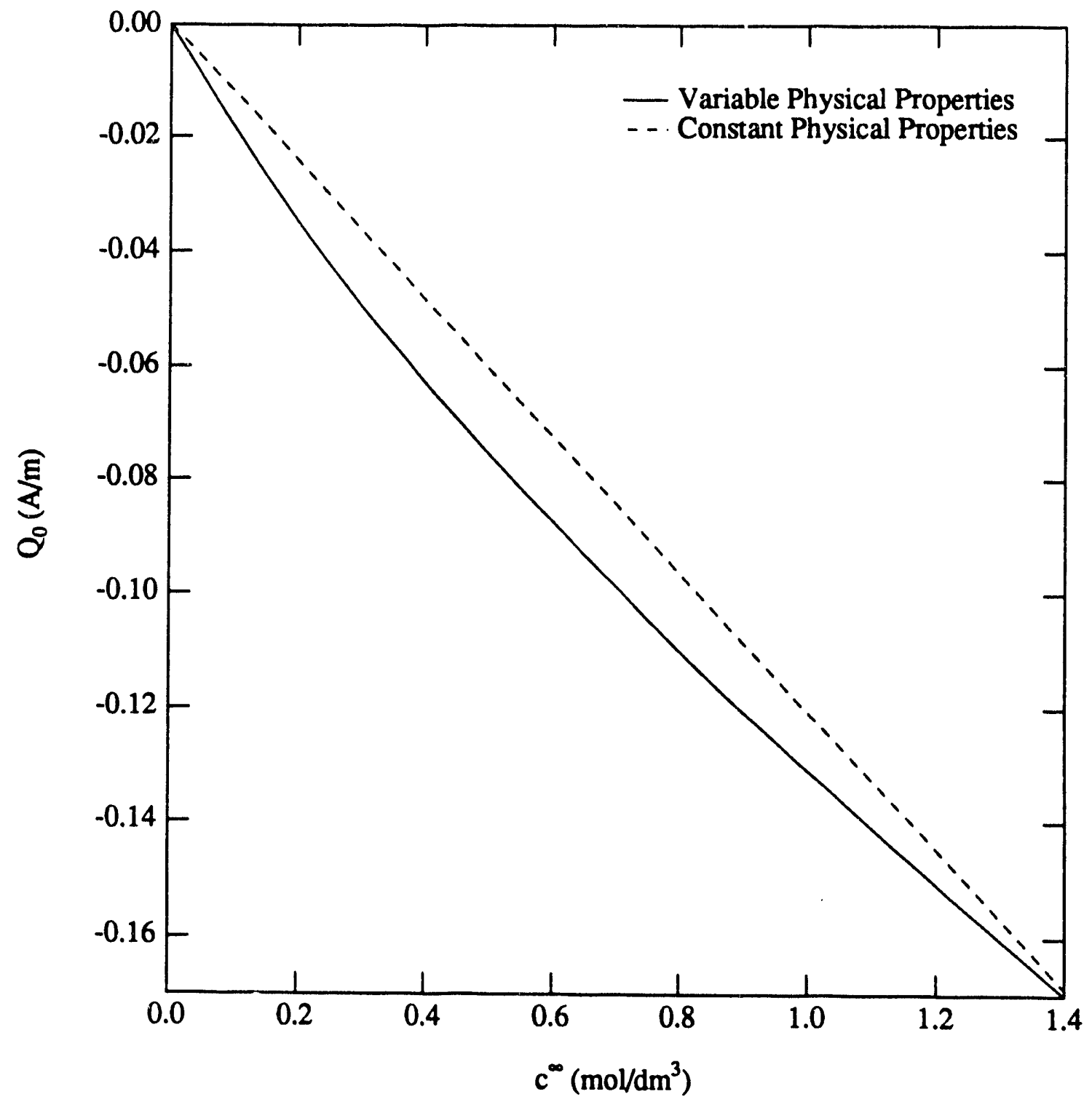

Figure 2. Quasi-potential at the surface of the electrode as a function of the concentration of $\mathrm{CuSO}_{4}$ in the bulk of the solution, in binary $\mathrm{CuSO}_{4}$ solution at limiting-current conditions. $i_{\text {avg }}=4 Q_{0} / \pi a$ for the disk, and $i_{\text {avg }}=Q_{0} / a$ for the hemisphere, where $a$ denotes the radius of the electrode. 


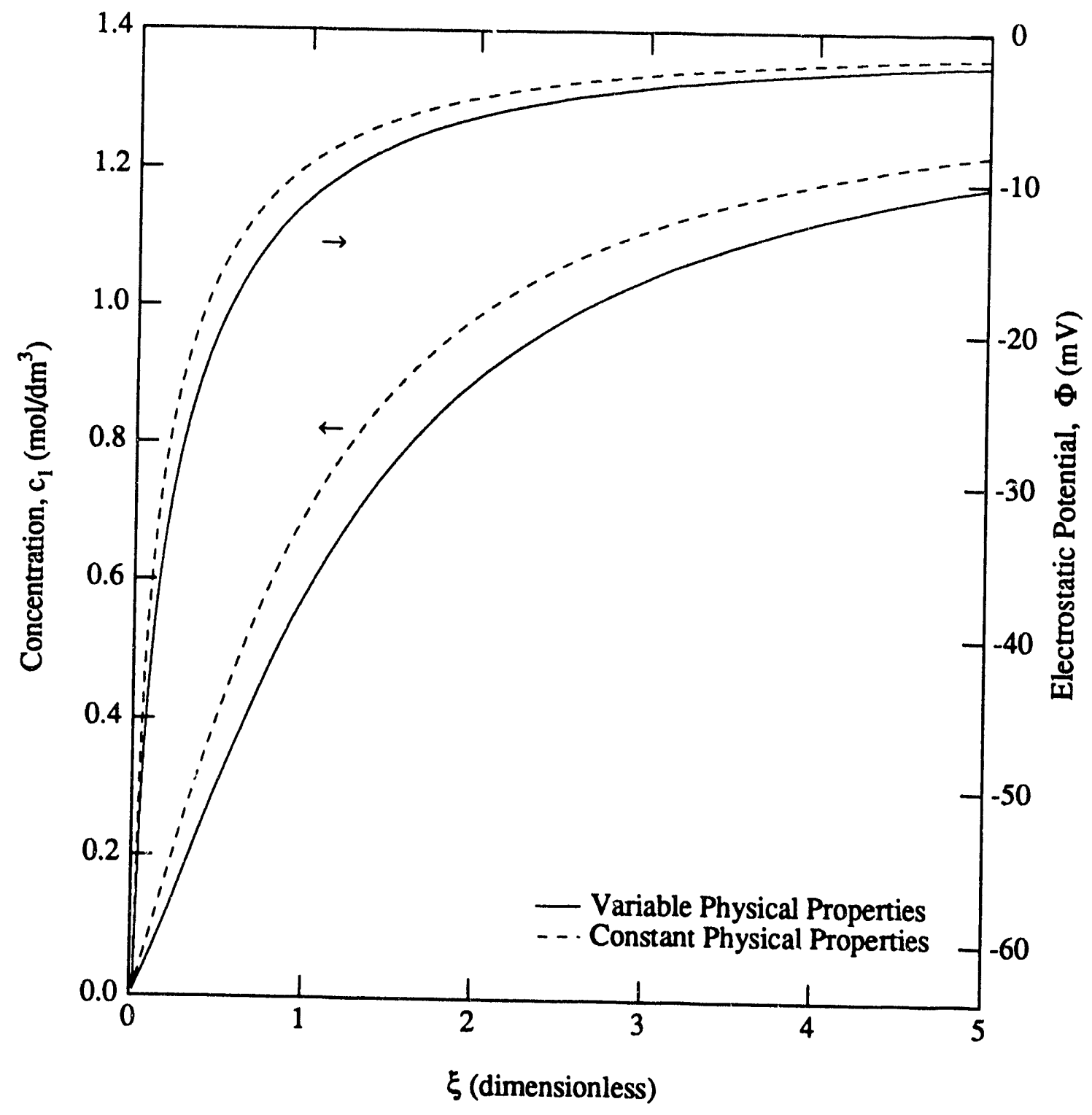

Figure 3. Electrostatic potential and concentration as functions of the rotational elliptic coordinate, $\xi$, in binary $\mathrm{CuSO}_{4}$ solution at limiting-current conditions, over a disk electrode. The bulk concentration of $\mathrm{CuSO}_{4}$ is $1.40 \mathrm{~mol} / \mathrm{dm}^{3}$. 


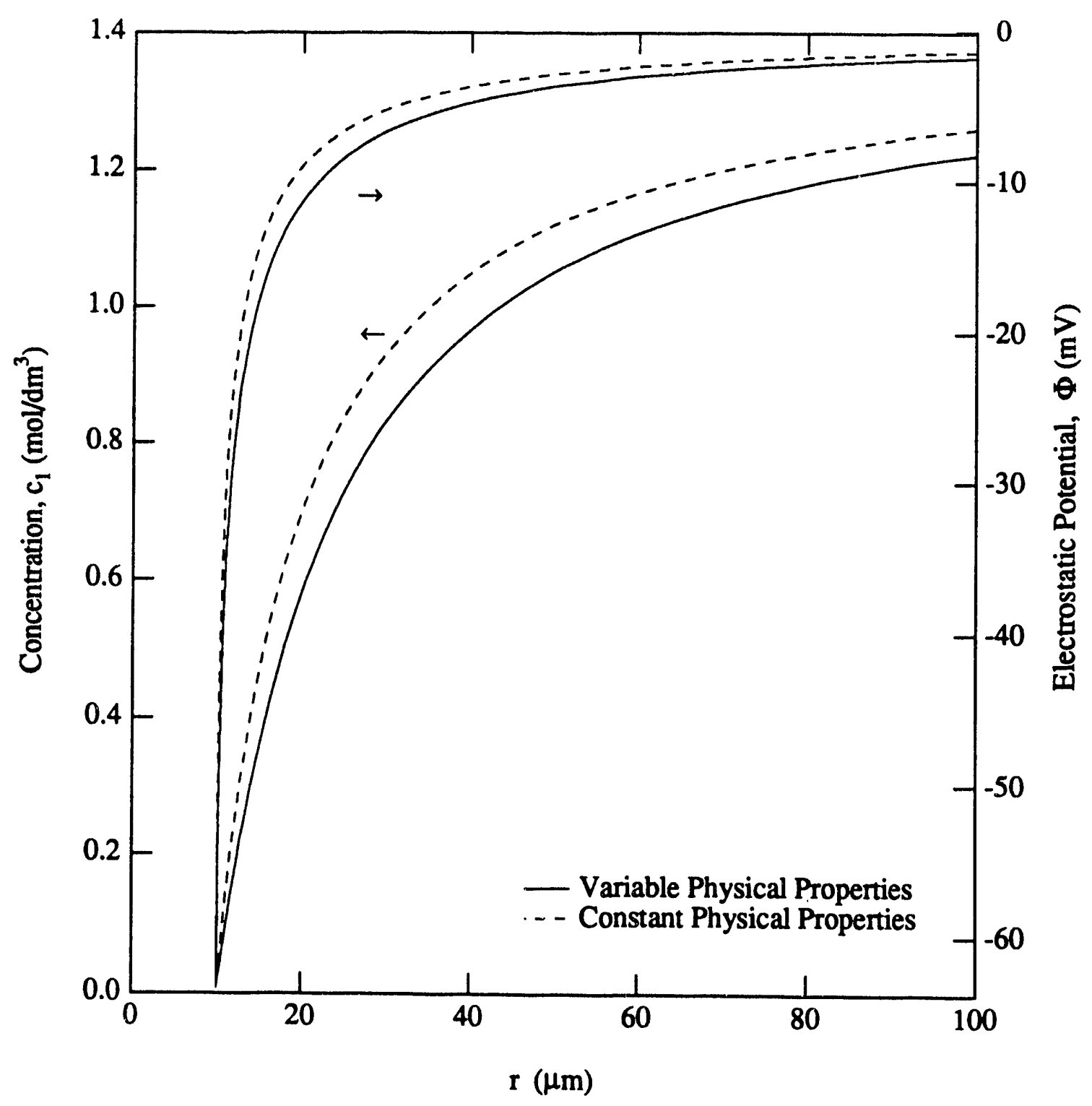

Figure 4. Electrostatic potential and concentration as functions of the radius, $r$, in binary $\mathrm{CuSO}_{4}$ solution at limiting-current conditions, over a hemisphere electrode of radius 10 $\mu \mathrm{m}$. The bulk concentration of $\mathrm{CuSO}_{4}$ is $1.40 \mathrm{~mol} / \mathrm{dm}^{3}$. 


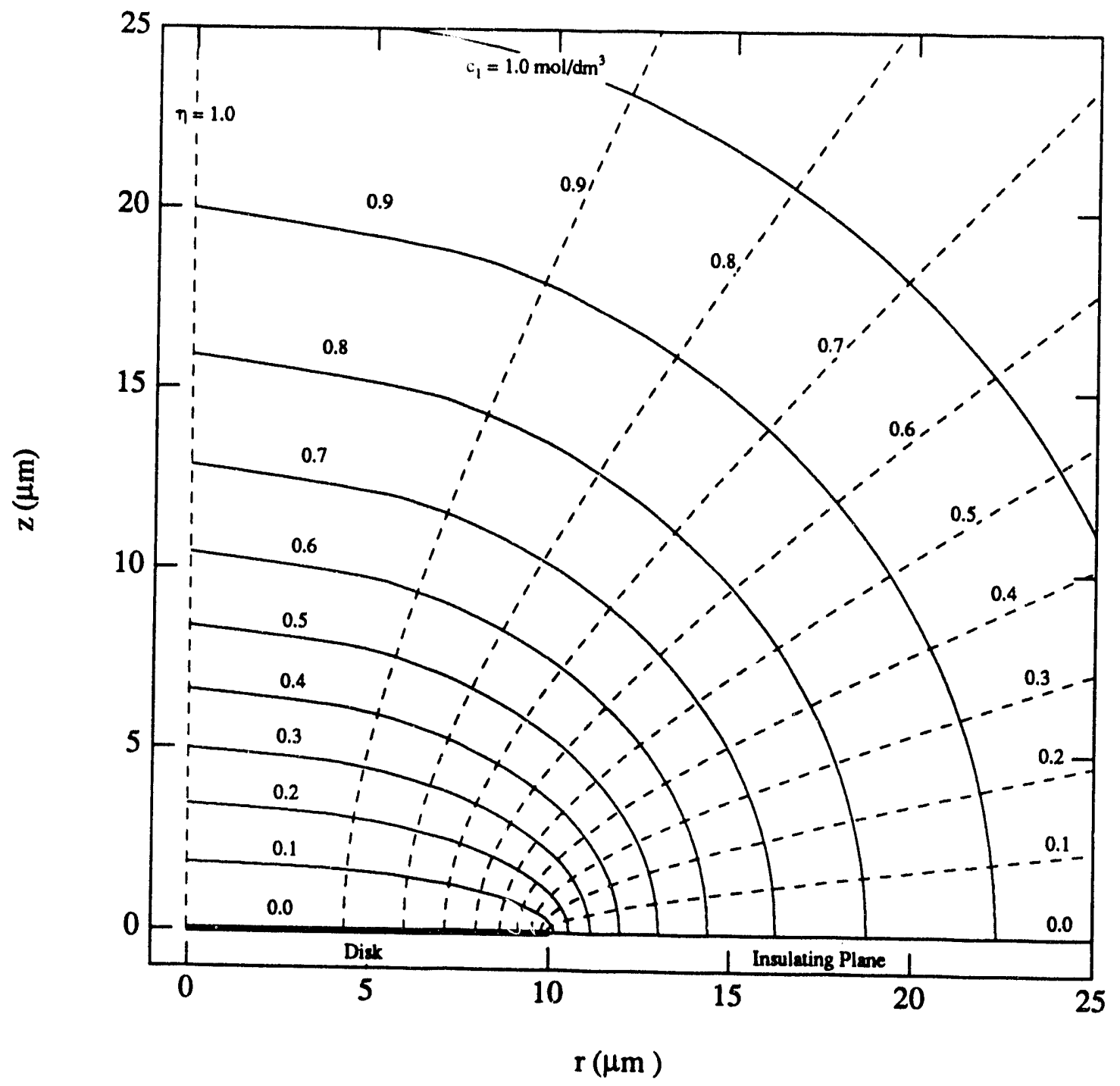

Figure 5. Equi-concentration lines and current-lines in binary $\mathrm{CuSO}_{4}$ solution at limitingcurrent conditions, over a disk electrode of radius $10 \mu \mathrm{m}$. The equi-concentration lines are also lines of constant electrostatic potential and quasi-potential. The bulk concentration of $\mathrm{CuSO}_{4}$ is $1.40 \mathrm{~mol} / \mathrm{dm}^{3}$. 


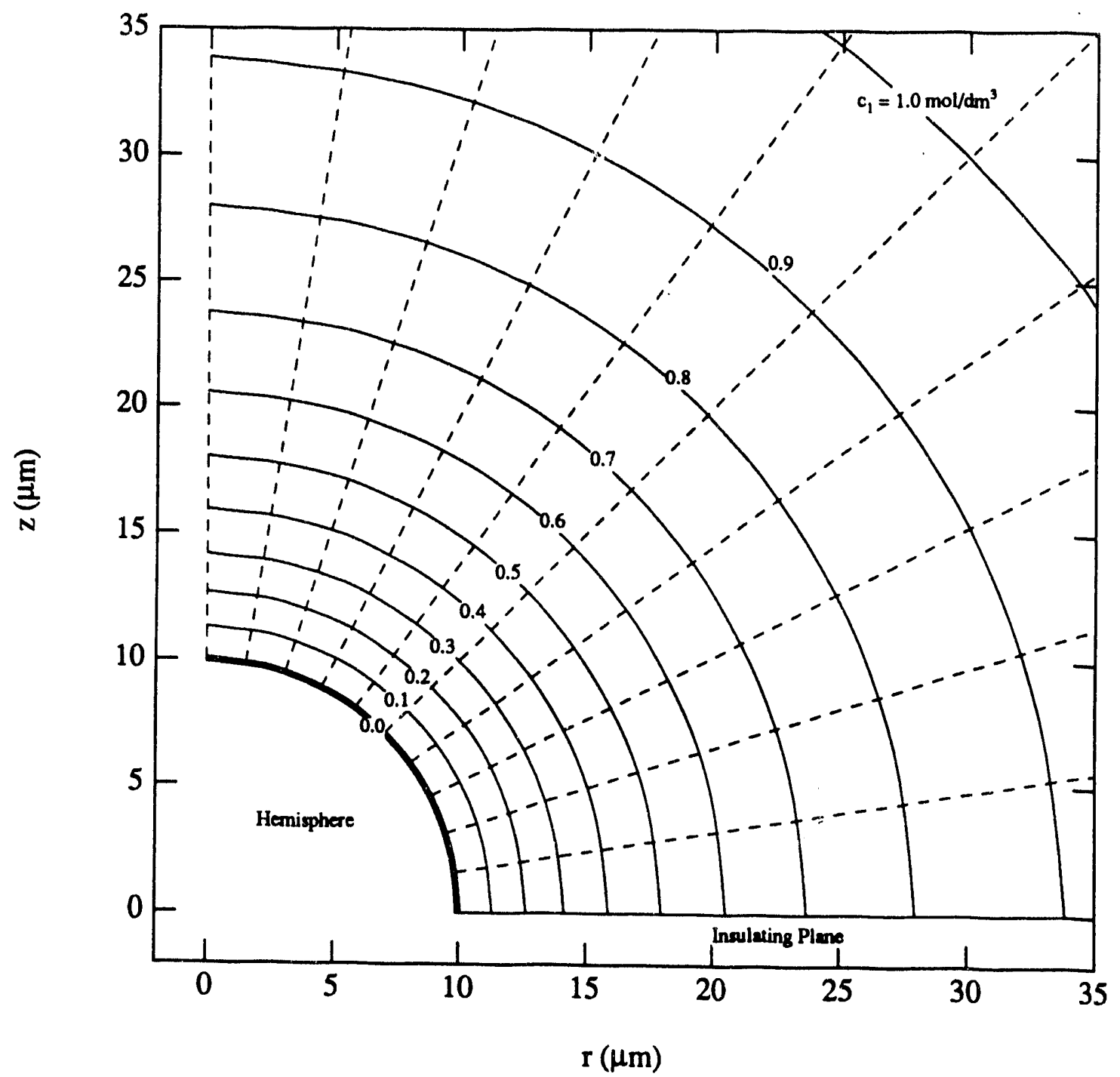

Figure 6. Equi-concentration lines and current-lines in binary $\mathrm{CuSO}_{4}$ solution at limitingcurrent conditions, over a hemisphere electrode of radius $10 \mu \mathrm{m}$. The equi-concentration lines are also lines of constant electrostatic potential and quasi-potential. The bulk concentration of $\mathrm{CuSO}_{4}$ is $1.40 \mathrm{~mol} / \mathrm{dm}^{3}$. 
For uniform-current-density distributions, one of the properties we are interested in is the variation of the electrostatic potential, adjacent to the electrode, just outside the double-layer. This is shown in figure 7 with the value of the current normal to the electrode set to various fractions of the average limiting-current density.

\subsection{Acidic Copper Sulfate}

We study this system to illustrate better the means of treating homogeneous reactions with the quasi-potential transformation. It also gives us an opportunity to see the effect of supporting electrolyte on the potential drop in the system. The deposition reaction has been discussed in section 5.0. The homogeneous reaction that must be considered here is the incomplete dissociation of bisulfate ions $\mathrm{HSO}_{4}^{-}$

$$
\mathrm{HSO}_{4}^{-} \rightleftarrows \mathrm{H}^{+}+\mathrm{SO}_{4}^{2-} \text {, }
$$

and we assume that this reaction is at equilibrium.

Young et al. [20] used Raman spectroscopy to measure the equilibria present in, among others, aqueous solutions of $\mathrm{H}_{2} \mathrm{SO}_{4}$. From their measurements, it would appear safe to assume that for solutions more dilute than $10 \mathrm{M}$, all of the $\mathrm{H}_{2} \mathrm{SO}_{4}$ is completely dissociated into $\mathrm{H}^{+}$, $\mathrm{HSO}_{4}^{-}$, and $\mathrm{SO}_{4}^{2-}$ ions, and that there is no $\mathrm{H}_{2} \mathrm{SO}_{4}$ present in the system in molecular form. ${ }^{\dagger}$ In keeping with the assumptions

$\dagger$ These authors could not measure the presence of any molecular 


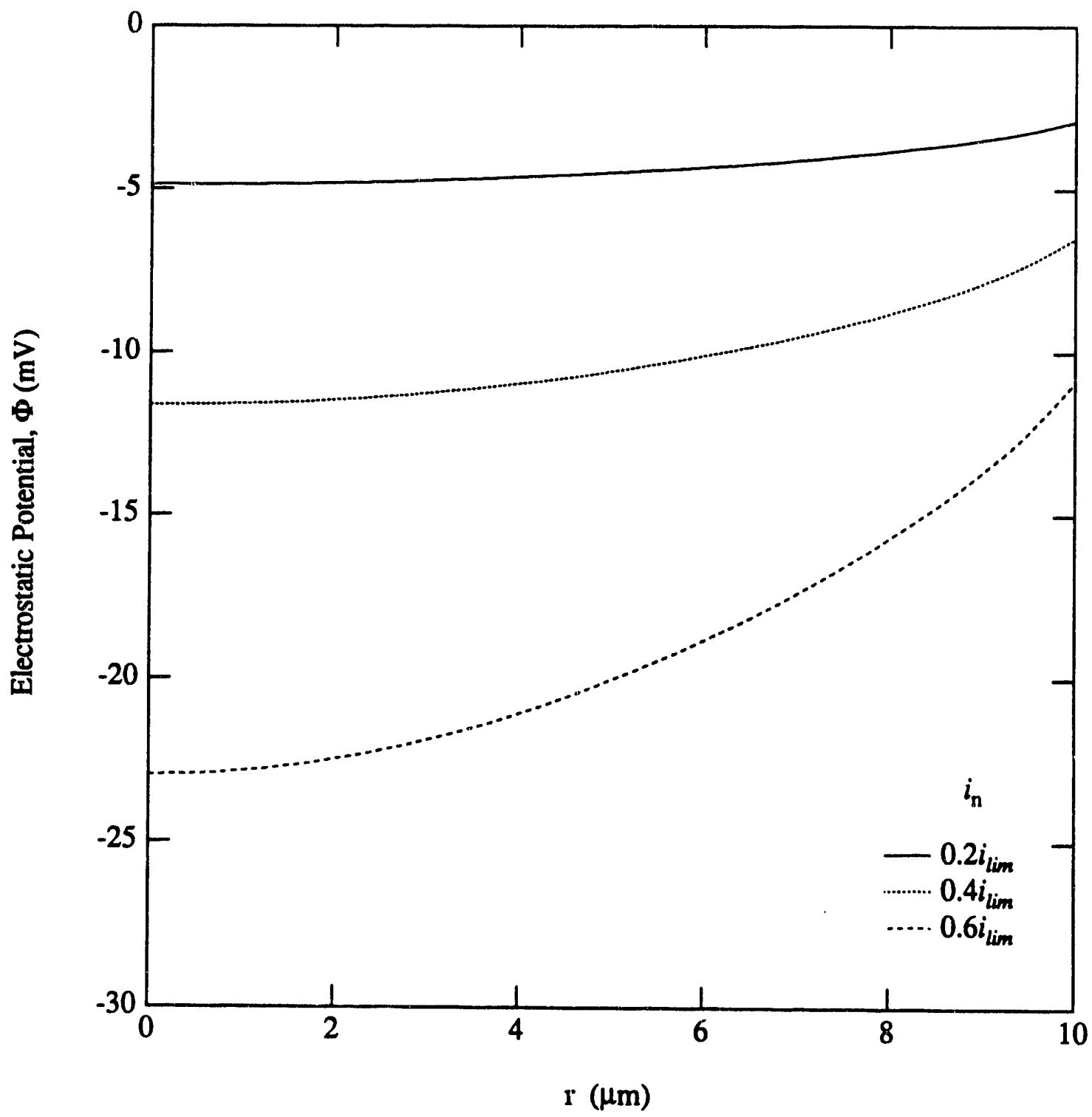

Figure 7. Variation of electrostatic potential adjacent to the surface of a disk electrode of radius $10 \mu \mathrm{m}$, in binary $\mathrm{CuSO}_{4}$ solution at uniform-current-density conditions. The limiting-current density is $-2.1630 \mathrm{~A} / \mathrm{cm}^{2}$. The bulk concentration of $\mathrm{CuSO}_{4}$ is 1.40 $\mathrm{mol} / \mathrm{dm}^{3}$. 
implicit in dilute-solution theory, we ignore the interactions between the ions in the solution and assume that the behavior of the $\mathrm{H}_{2} \mathrm{SO}_{4}$ is the same when $\mathrm{CuSO}_{4}$ is present.

The mass transfer in this system has been investigated previously for the rotating disk and diffusion cell configurations by Hsueh and Newman [21]. They used the following relationship between the apparent dissociation constant

$$
K^{\prime}=\frac{{ }_{\mathrm{H}^{+}}{ }^{c} \mathrm{SO}_{4}^{2-}}{{ }_{\mathrm{HSO}_{4}^{-}}}
$$

and the thermodynamic dissociation constant

$$
\begin{gathered}
K=K \cdot \frac{f}{\frac{f}{\mathrm{HSO}_{4}^{+}}{ }^{f} \mathrm{SO}_{4}^{2-}}, \\
\ln \left(K^{\prime} / K\right)=\frac{5.29 I_{r}^{\frac{1}{2}}}{1+0.56 I_{r}^{\frac{1 / 2}{2}}}
\end{gathered}
$$

where $K=0.0104 \mathrm{~mol} . \mathrm{dm}^{-3}$ and $I_{r}$ is the true ionic strength,

$$
I_{r}=\frac{1}{2} \sum_{i} z_{i}^{2} c_{i}
$$

Hsueh and Newman assumed that $K^{\prime}$ remained constant with respect to ionic strength, and hence with respect to position, and assigned to it its value at infinity. In this work, we allow $K^{\prime}$ to vary with $\mathrm{H}_{2} \mathrm{SO}_{4}$ at concentrations less than $14 \mathrm{M}$. They do point out that this may be due to the overshadowing of the $910 \mathrm{~cm}^{-1}$ line, and that $\mathrm{H}_{2} \mathrm{SO}_{4}$ may exist in molecular form in systems as dilute as $12 \mathrm{M}$, or even 10 $M$. This is not of particular concern, since the systems we work with are around $2 \mathrm{M}$. 
ionic strength, which varies with position; or more specifically, with electrostatic potential. In some of the results presented here we shall show the effect of assuming $K^{\prime}$ to be constant with respect to position.

In this system we ignore the effects of variations in the transport properties. As is evident, there are two concentrations that can be independently altered within the limits of $\mathrm{CuSO}_{4}$ solubility, these being the concentration of $\mathrm{H}_{2} \mathrm{SO}_{4}$ and $\mathrm{CuSO}_{4}$ in the bulk solution. There are four ionic species present, and the diffusion coefficient of each species depends on the interaction of that species with the other three and the solvent. One could speculate on using a method similar to that used to correlate the dissociation constant; by accounting for the effect on the activity of each ion due to the presence of the other ions by a correlation based on the ionic strength. However, the viscosity also plays an important role in determining the value of the diffusion coefficient. The level of complexity that would be introduced by accounting for the viscosity as well as the activity changes would defeat the purpose of using dilute-solution theory instead of concentrated-solution theory. This case illustrates the ambiguities inherent in using dilute-solution theory with any solution other than a binary solution, which is a special case in that the equation governing mass transfer derived from dilute-solution theory is similar in form to that derived using concentrated-solution theory (see Newman [5]). As mentioned previously, it is believed that the quasi-potential transformation can be 
used with concentrated-solution theory, and that has been left to future work.

The diffusion coefficients to be used in this work are taken from Newman [5], and are those at infinite dilution

\begin{tabular}{|c|l|c|}
\hline$i$ & species & $D_{i}\left(10^{-5} \mathrm{~cm}^{2} / \mathrm{s}\right)$ \\
\hline 1 & $\mathrm{Cu}^{2+}$ & 0.7188 \\
2 & $\mathrm{SO}_{4}^{2-}$ & 1.065 \\
3 & $\mathrm{H}^{+}$ & 9.312 \\
4 & $\mathrm{HSO}_{4}^{-}$ & 1.331 \\
\hline
\end{tabular}

The one further physical consideration is that the solubility of $\mathrm{CuSO}_{4}$ decreases with increasing $\mathrm{H}_{2} \mathrm{SO}_{4}$ concentration. The solubility data of Goodwin and Horsch [22] indicates that the solubility of $\mathrm{CuSO}_{4}$ is at least $0.9 \mathrm{~mol} \cdot \mathrm{dm}^{-3}$ at a $\mathrm{H}_{2} \mathrm{SO}_{4}$ concentration of 1.50 mol. $\mathrm{dm}^{-3}$ and goes up to $1.4083 \mathrm{~mol} . \mathrm{dm}^{-3}$ at zero $\mathrm{H}_{2} \mathrm{SO}_{4}$ concentration at $25^{\circ} \mathrm{C}$. In this work, the results we show are for a $\mathrm{CuSO}_{4}$ bulk concentration of $0.50 \mathrm{~mol} . \mathrm{dm}^{-3}$ and $\mathrm{a} \mathrm{H}_{2} \mathrm{SO}_{4}$ bulk concentration of 1.50 $\mathrm{mol} \cdot \mathrm{dm}^{-3}$.

The material balance equation for species 2,3 , and 4 are

$$
\begin{aligned}
& -\nabla \cdot N_{2}+R=0, \\
& -\nabla \cdot N_{3}+R=0,
\end{aligned}
$$

and 


$$
-\nabla \cdot N_{4}-R=0
$$

Adding and combining, we get

$$
\nabla \cdot\left(\mathrm{N}_{2}+\mathrm{N}_{4}\right)=0
$$

and

$$
\nabla \cdot\left(\mathrm{N}_{3}+\mathrm{N}_{4}\right)=0
$$

and by the theorem of Gibbs, since the normal components of $\mathrm{N}_{2}, \mathrm{~N}_{3}$, and $\mathrm{N}_{4}$ are 0 at all finite boundaries,

$$
\mathrm{N}_{2}+\mathrm{N}_{4}=0
$$

and

$$
\mathrm{N}_{3}+\mathrm{N}_{4}=0
$$

everywhere. Substituting equations 12 and 13 we find

$$
-D_{2}\left[-2 c_{2}+\frac{d c_{2}}{d \phi}\right]-D_{4}\left[-c_{4}+\frac{d c_{4}}{d \phi}\right]=0
$$

and

$$
-D_{3}\left[c_{3}+\frac{d c_{3}}{d \phi}\right]-D_{4}\left[-c_{4}+\frac{d c_{4}}{d \phi}\right]=0 .
$$

The equilibrium relationship is equation 28

$$
K^{\prime}=\frac{c_{2} c_{3}}{c_{4}} .
$$

These three equations, together with the equation of electroneutrality and equation 14 (after substituting equation 13) constitute a set of five first-order, ordinary differential equations for the five unknowns $\left(c_{1}, c_{2}, c_{3}, c_{4}\right.$, and $\left.Q\right)$ in the electrostatic potential $\phi$. The initial values required to solve for the unknowns are their 
values at infinity, where $\phi$ is zero. These values are obtained from a root-finding procedure, utilizing Newton's method, from which we find $K^{\prime}$ and all the concentrations at infinity. This set of equations has been solved by finding the first derivatives (we need to differentiate the equation of electroneutrality) and using the fourth order Runge-Kutta algorithm. The computer program used to solve this problem is shown in Appendix A.

Figure 8 shows the values of the concentrations and the electrostatic potential as functions of the quasi-potential. Figure 9 shows the value of the quasi-potential at the surface of the electrode as a function of the bulk concentration of $\mathrm{CuSO}_{4}$, for various values of bulk concentration of $\mathrm{H}_{2} \mathrm{SO}_{4}$. Since we chose the electrostatic potential to be the independent variable in the system-specific calculations, it became difficult to evaluate the quasi-potential at the surface for a bulk $\mathrm{H}_{2} \mathrm{SO}_{4}$ concentration of zero, as the electrostatic potential at the surface is infinite in a binary electrolyte at limiting-current conditions. As with figure 2, these values can be used to obtain the values of the average limiting current.

Figure 10 and figure 11 show the results of the combination of the system-specific calculations shown in figure 8 with the results of the geometry-specific calculations (equations 21 and 26) for the limiting-current distributions. They show the various concentrations and electrostatic potential as functions of the spatial variables. Shown also are the consequences of holding the dissociation constant constant with respect to position. We have not shown plots of these 


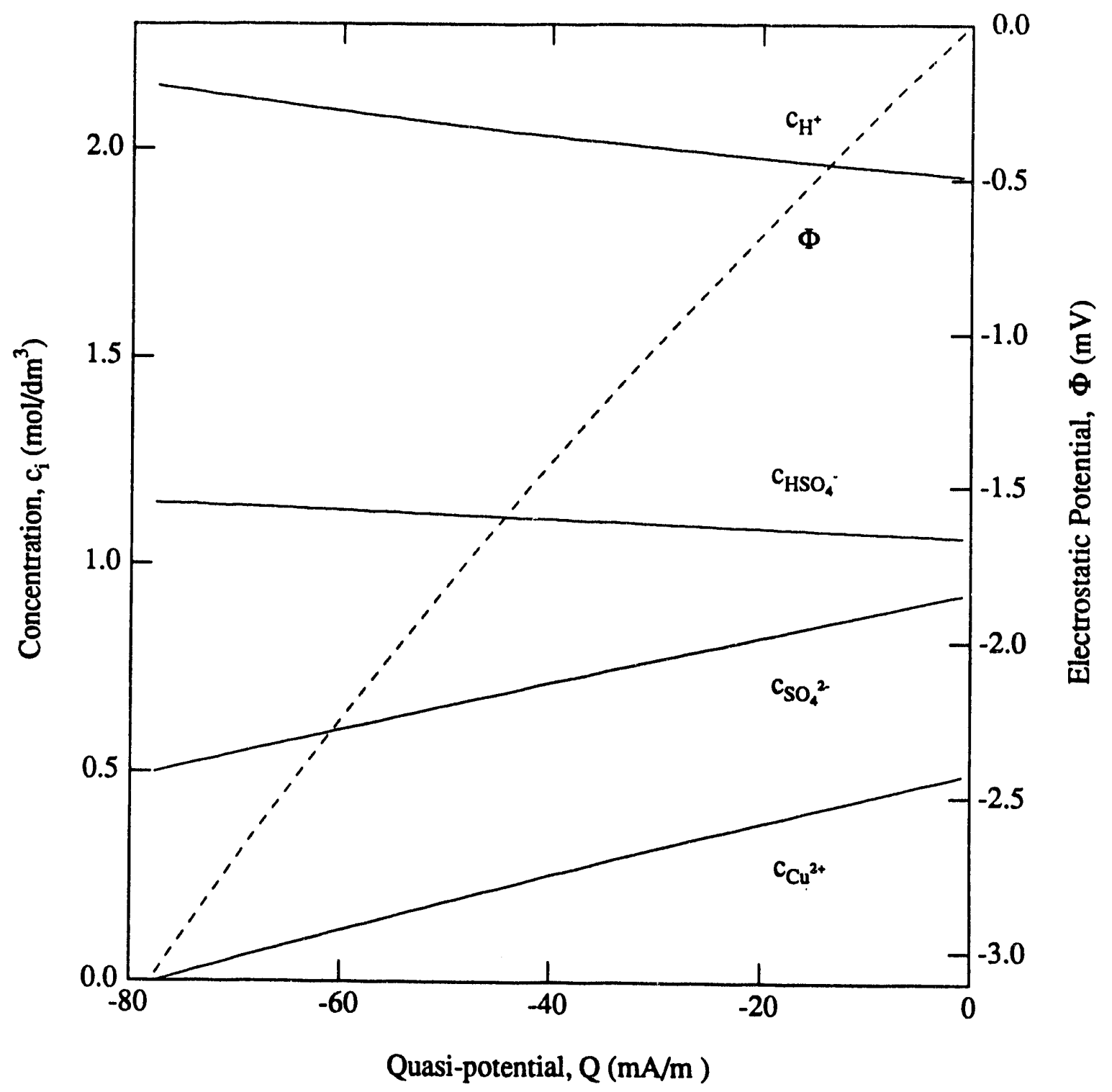

Figure 8. Electrostatic potential and ionic concentrations as functions of the quasi-potential in acidic $\mathrm{CuSO}_{4}$ solution. The bulk concentration of $\mathrm{CuSO}_{4}$ is $0.50 \mathrm{~mol} / \mathrm{dm}^{3}$, and that of $\mathrm{H}_{2} \mathrm{SO}_{4}$ is $1.50 \mathrm{~mol} / \mathrm{dm}^{3}$. 


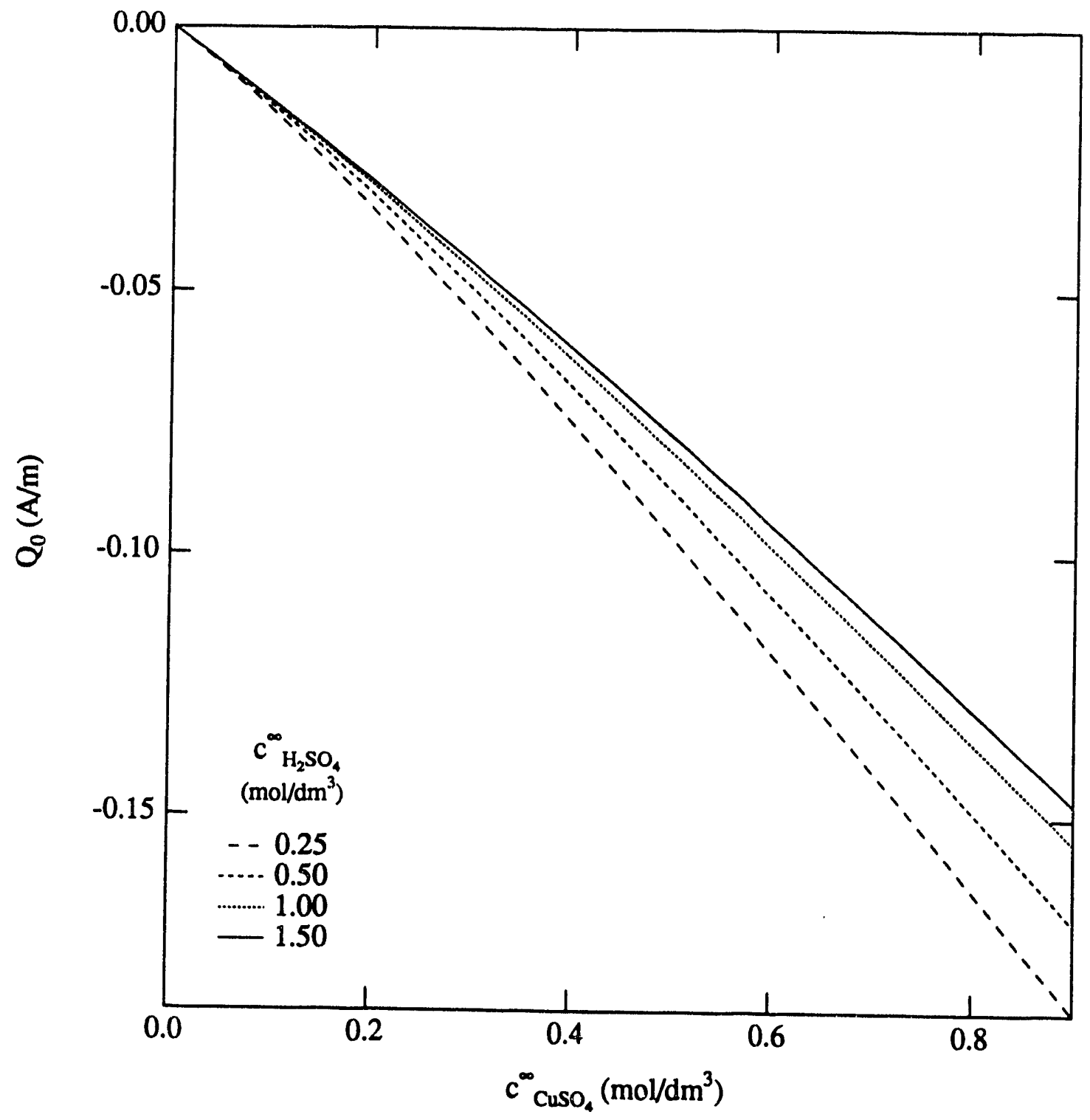

Figure 9. Quasi-potential at the surface of the electrode as a function of the concentration of $\mathrm{CuSO}_{4}$ in the bulk of the solution, in acidic $\mathrm{CuSO}_{4}$ solution at limiting-current conditions, for varying values of bulk $\mathrm{H}_{2} \mathrm{SO}_{4}$ concentration. $i_{\text {avg }}=4 Q_{0} / \pi a$ for the disk, and $i_{\text {avg }}=Q_{0} / a$ for the hemisphere, where $a$ denotes the radius of the electrode. 


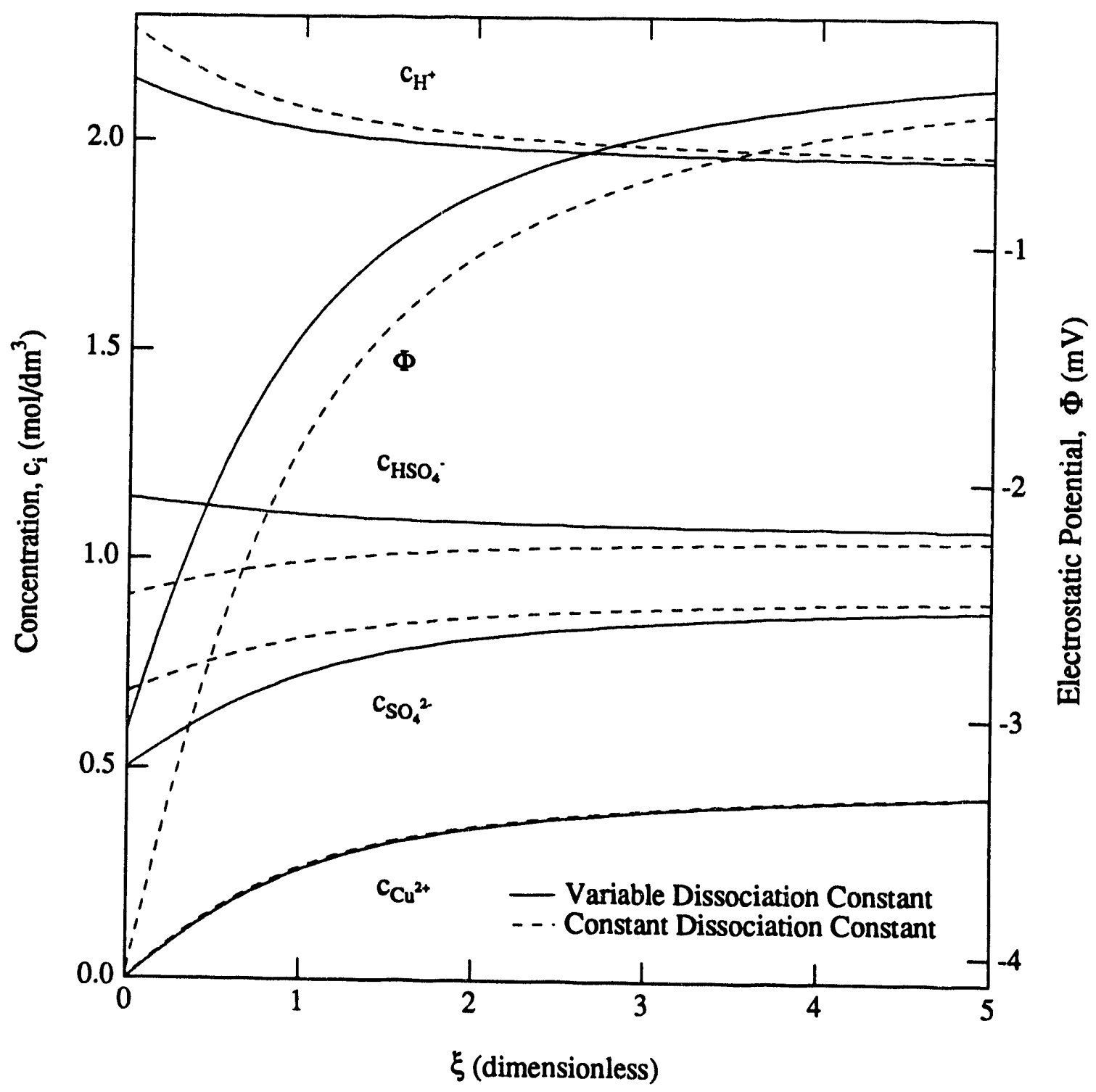

Figure 10. Electrostatic potential and ionic concentrations as functions of the rotational elliptic coordinate, $\xi$, in acidic $\mathrm{CuSO}_{4}$ solution at limiting-current conditions, over a disk electrode, showing the effect of assuming the dissociation constant to be constant with respect to position. The bulk concentration of $\mathrm{CuSO}_{4}$ is $0.50 \mathrm{~mol} / \mathrm{dm}^{3}$, and that of $\mathrm{H}_{2} \mathrm{SO}_{4}$ is $1.50 \mathrm{~mol} / \mathrm{dm}^{3}$. 


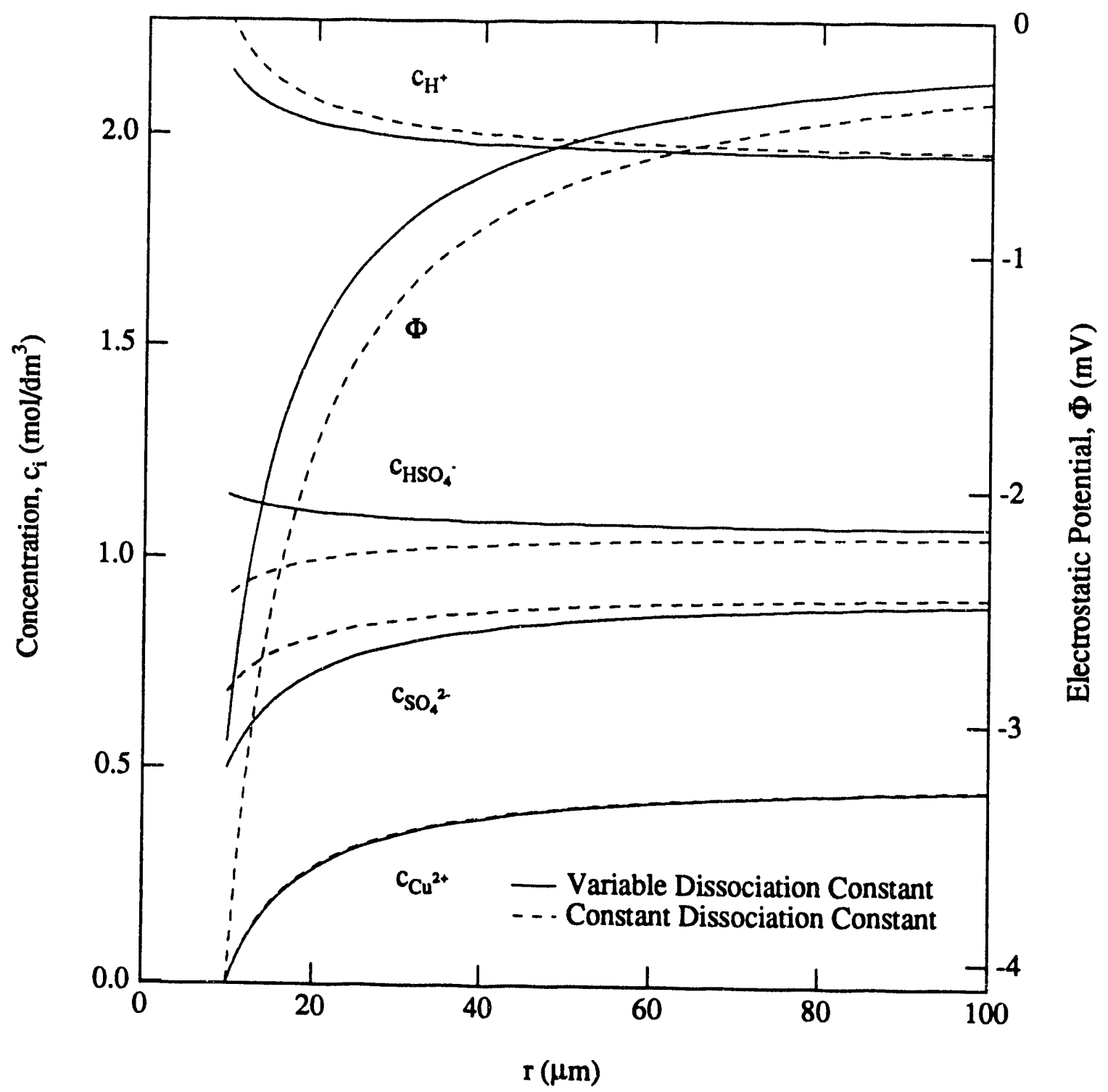

Figure 11. Electrostatic potential and ionic concentrations as functions of the radius, $r$, in acidic $\mathrm{CuSO}_{4}$ solution at limiting-current conditions, over a hemisphere electrode of radius $10 \mu \mathrm{m}$, showing the effect of assuming the dissociation constant to be constant with respect to position. The bulk concentration of $\mathrm{CuSO}_{4}$ is $0.50 \mathrm{~mol} / \mathrm{dm}^{3}$, and that of $\mathrm{H}_{2} \mathrm{SO}_{4}$ is $1.50 \mathrm{~mol} / \mathrm{dm}^{3}$. 
concentrations and the current lines as functions of the cylindrical coordinates as we did in figures 5 and 6 , since we expect them to look exactly the same as those figures with changes in the values of concentration or potential at the equi-potential lines.

Figure 12 shows the variation of the electrostatic potential adjacent to the disk electrode at uniform-current-density conditions, for various fractions of the average limiting-current density. Except for the change of scale, this figure is virtually identical to figure 7 for the binary $\mathrm{CuSO}_{4}$ system. The curve that represents the case of the normal component of the current density being set to 0.785 of the average limiting-current density represents also the maximum current density that can be passed at unform-current-density conditions.

\subsection{Effect of Migration on Limiting Currents}

Newman [5] has discussed in detail the effect of taking into account the contribution to the mass transfer by migration. A simple measure of the effect of migration is the ratio of the limiting current calculated with consideration of migration to the diffusionlimiting current, which is the limiting current evaluated with diffusion considered to be the only mechanism of mass transfer. ${ }^{\dagger}$

As we have seen for both the disk and hemisphere electrodes, the limiting current density normal to the electrode, for any system in

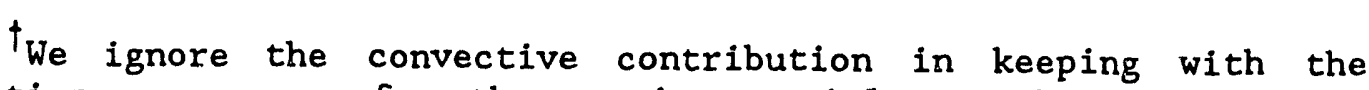
conditions necessary for the quasi-potential transformation to be applicable. 


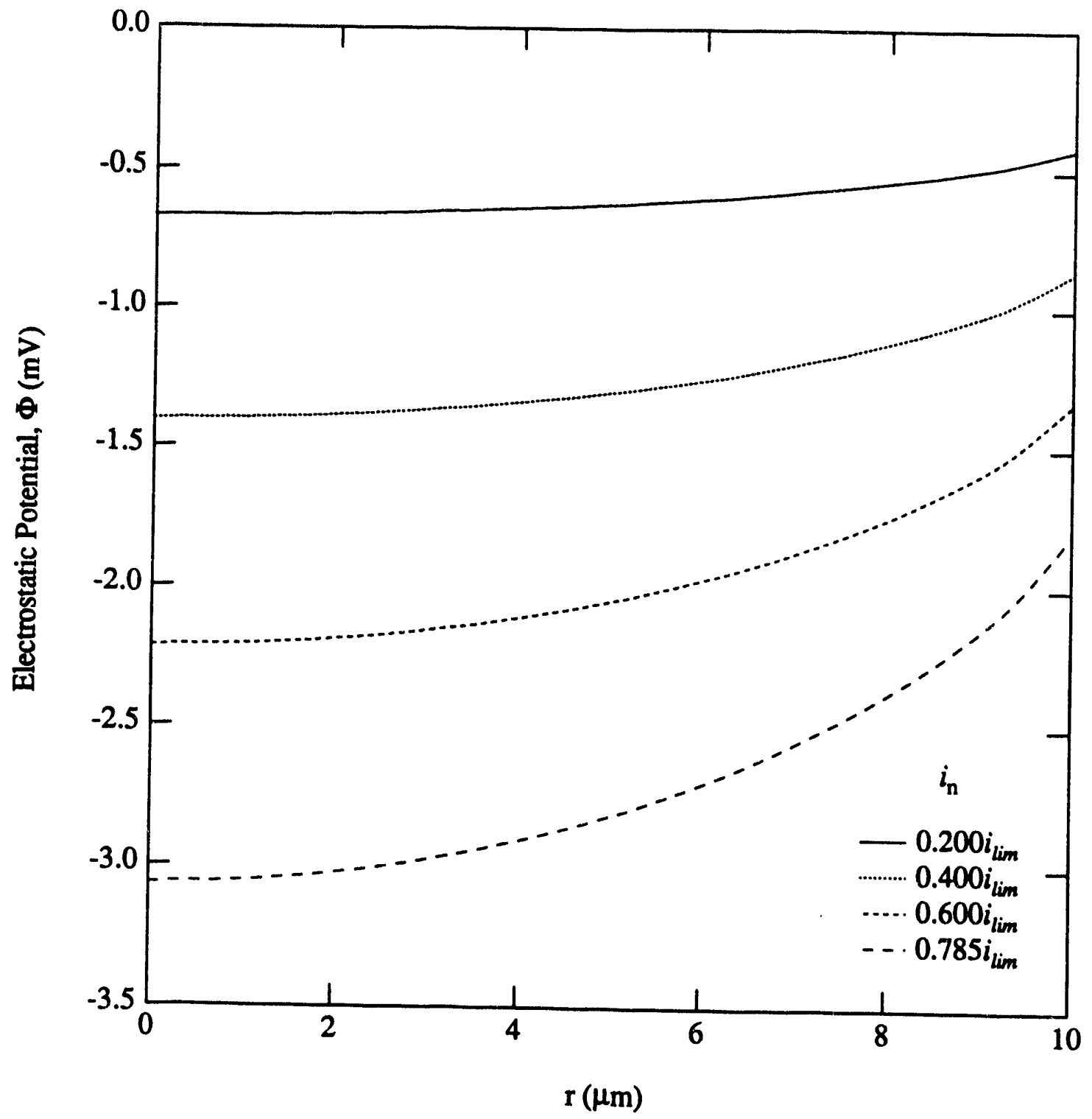

Figure 12. Variation of electrostatic potential adjacent to the surface of a disk electrode of radius $10 \mu \mathrm{m}$, in acidic $\mathrm{CuSO}_{4}$ solution at uniform-current-density conditions. The limiting current density is $-0.98462 \mathrm{~A} / \mathrm{cm}^{2}$. The bulk concentration of $\mathrm{CuSO}_{4}$ is $0.50 \mathrm{~mol} / \mathrm{dm}^{3}$, and that of $\mathrm{H}_{2} \mathrm{SO}_{4}$ is $1.50 \mathrm{~mol} / \mathrm{dm}^{3}$. 
which the quasi-potential transformation is applicable, takes the form

$$
i_{L}=Q_{0} g(\mathbf{x})
$$

where $g(x)$ is a function of the geometry of the system and is obtained from the geometry-specific calculation. The subscript $L$ indicates that the property is evaluated at limiting-current conditions. We see, obviously, that

$$
i_{L D}=Q_{D O} g(x)
$$

where the subscript $D$ indicates that the property is evaluated with diffusion considered to be the only mechanism of mass transfer. This function $g(x)$, appearing in the expression for the diffusion-limiting current, is identical to that appearing in the expression for the limiting current, since it is independent of the system-specific part of the calculation. It follows that

$$
\frac{i_{L}}{i_{L D}}=\frac{Q_{0}}{Q_{D O}}
$$

for all systems in which the quasi-potential transformation is applicable. Since the value of the quasi-potential on the surface of the electrode is a result of the system-specific calculation only, the effect of migration on the limiting current, written in this form, is independent of the geometry of the system.

In deriving the expression above for the diffusion-limiting current, we have defined the quasi-potential for the diffusion-only case in the same manner as we did for the case in which diffusion and 
migration are considered:

$$
1_{D}=-\nabla Q_{D} .
$$

For the diffusion-only case, the equation for the flux of the principal reactant (equation 5 ) reduces to

$$
\mathrm{N}_{R D}=-D_{R} \nabla c c_{R}
$$

Faraday's law states that

$$
N_{R n}=-\frac{s_{R}}{n F} i_{n},
$$

at the electrode surface. We know that

$$
\nabla \cdot 1=0
$$

and from the material balance for species $R$, if $R$ does not participate in any homogeneous reaction,

$$
\nabla \cdot \mathrm{N}_{R}=\mathrm{J}
$$

Since each vector is individually solenoidal, we write that

$$
\nabla \cdot\left(\mathrm{N}_{R}+\frac{s_{R}}{n F} 1\right)=0 .
$$

From the theorem of Gibbs and Faraday's Law, it follows that

$$
\mathrm{N}_{R}+\frac{s_{R}}{n F} 1=0,
$$

everywhere. Substituting for $N_{R D}$ and $I_{D}$ gives

$$
-D_{R} \nabla c_{R}-\frac{s_{R}}{n F} \nabla Q_{D}=0 .
$$

Therefore we find that 


$$
\frac{d Q_{D}}{d c_{R}}=-\frac{n F D_{R}}{s_{R}}
$$

For binary electrolyte systems we can replace the equation for $D_{R}$ by the expression involving the salt diffusion coefficient and the transference number of the principal reactant, given in section 2 . Upon substituting, integrating, applying the boundary condition at infinity, and setting the concentration of the principal reactant to zero, its concentration at the electrode surface, we find for binary electrolyte systems

$$
Q_{D 0}=\frac{-n}{s_{R}} \frac{z_{2}}{\left(z_{2}-z_{R}\right)} F \int_{c_{R}^{\infty}}^{0} \frac{D}{\left(1-t_{R}\right)} d c_{R}
$$

where the subscript 2 refers to the counterion. From equation 19, for binary electrolyte systems

$$
Q_{0}=z_{R} F \int_{c_{R}^{\infty}}^{0} \frac{D}{\left(1-t_{R}\right)} d c_{R}
$$

It follows that

$$
\frac{i_{L}}{i_{L D}}=\frac{-s_{R}}{n} \frac{z_{R}\left(z_{2}-z_{R}\right)}{z_{2}},
$$

for binary electrolyte systems. For the binary $\mathrm{CuSO}_{4}$ solution, specifically,

$$
\frac{i_{L}}{i_{L D}}=2
$$

For systems in which there are multiple electrolyte species present, we can obtain a general expression for the diffusionlimiting current if the transport properties are considered to be 
constant. Integrating the differential equation for $Q_{D}$, applying the boundary condition at infinity, and setting the concentration of the principal reactant to zero, we find

$$
Q_{D 0}=\frac{n F D_{R}}{s_{R}} c_{R}^{\infty} \text {. }
$$

It follows that for the acidic $\mathrm{CuSO}_{4}$ system, specifically,

$$
\frac{i_{L}}{i_{L D}}=\frac{Q_{0}}{-2 F D_{R} c_{R}^{\infty}},
$$

where $c_{R}$ is the concentration of cupric ions. In section 6.0 we discussed the method used in determining $Q_{0}$. We have two independent parameters in the system - the bulk concentrations of sulfuric acid and copper sulfate. The parameter $r$ used by Newman,

$$
r=\frac{c_{\mathrm{H}_{2} \mathrm{SO}_{4}}^{\infty}}{c_{\mathrm{CuSO}_{4}}^{\infty}+c_{\mathrm{H}_{2} \mathrm{SO}_{4}}^{c}}
$$

gives an indication of how well a solution is supported. In figure 13 we show the effect of migration as a function of the square root of $r$, for various values of $\mathrm{CuSO}_{4}$ concentration. It is desirable to use the square root of $r$ since the effect of adding a little supporting electrolyte is very large.

Newman presents results (figure 19.2, page 402) showing the effect of migration on limiting currents in the Nernst stagnant diffusion layer, for the acidic $\mathrm{KCl}$ system. Since the Nernst stagnant diffusion layer is one of the geometries in which the quasi-potential transformation is applicable, this curve could easily be derived 


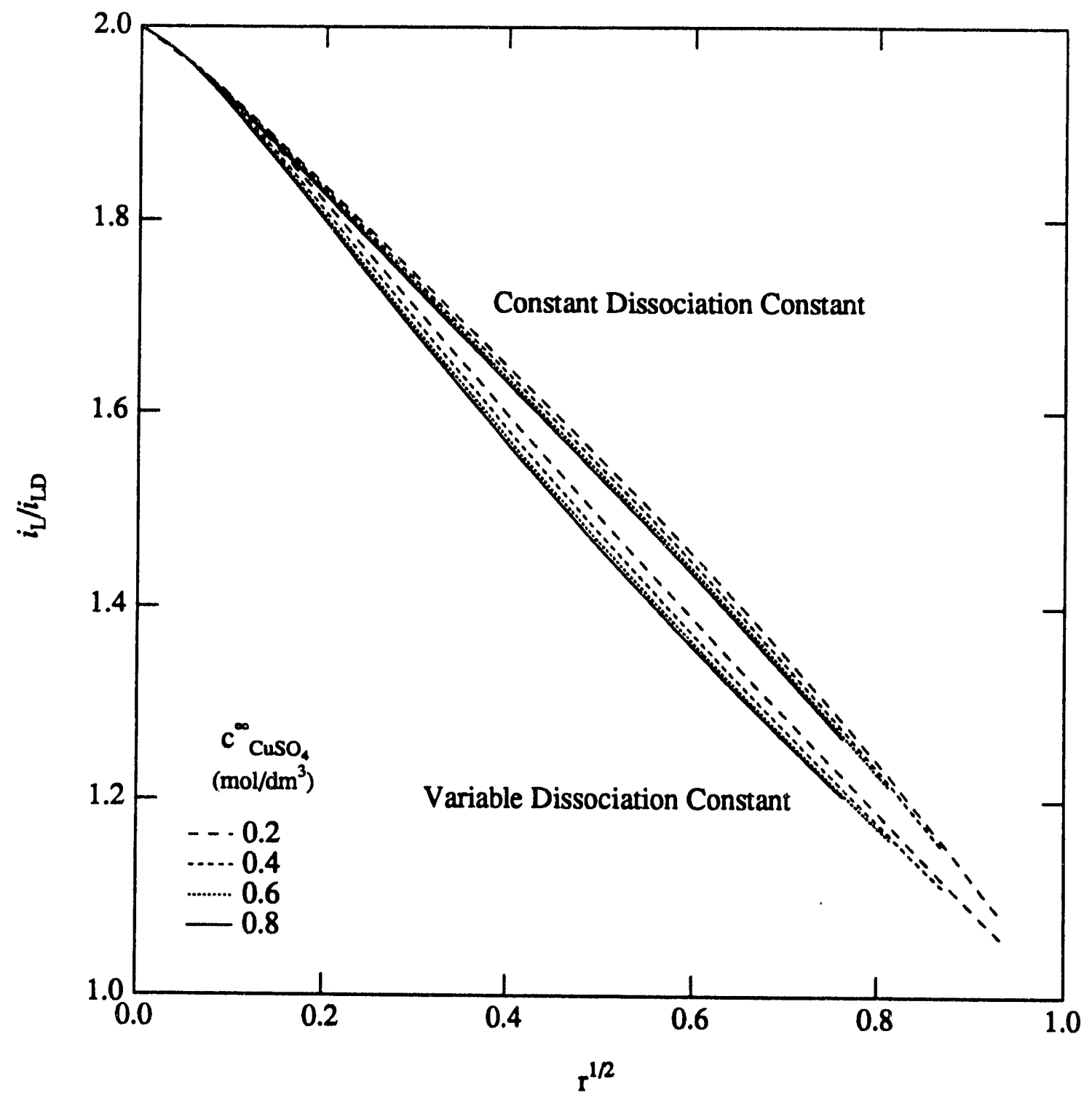

Figure 13. The effect of migration on limiting currents for the acidic $\mathrm{CuSO}_{4}$ system, shown for various values of the bulk concentration of $\mathrm{CuSO}_{4}$. The bulk concentration of $\mathrm{H}_{2} \mathrm{SO}_{4}$ is constrained by the limit of solubility. Also displayed is the effect of considering the dissociation constant to be constant with respect to position. 
using similar methods to those used for the acidic $\mathrm{CuSO}_{4}$ system. Newman also presents results (figure 19.13, page 415) for the acidic $\mathrm{CuSO}_{4}$ system for various values of the ratio of the bulk ionic strength at complete bisulfate dissociation, to the dissociation constant evaluated at bulk conditions. Since the dissociation constant is not held constant in our system, this parameter is not really relevant, and it is difficult to make a direct comparison of the results. For all of the curves shown in figure 13, this parameter assumes values between 2.72 and 4.72 ; this gives an indication of where our system lies in the parameter space of Newman's figure 19.13.

Newman also shows the change of concentration of the supporting electrolyte between the bulk and the surface. In terms of the quasi-potential transformation, this can be interpreted as a result of the system-specific part of the problem and independent of the geometry. Thus, for all geometries satisfying the results of the quasi-potential transformation, Newman's results for the Nernst stagnant diffusion layer apply here and axe different from those for two other classes of systems-the systems of the Lighthill transformation exemplified by the rotating-disk results and the transient systems (of the penetration model) exemplified by the results for a growingmercury drop and for transient transport in a semi-infinite stagnant diffusion layer. 


\section{Summary}

The quasi-potential transformation transforms the set of coupled second-order, nonlinear partial differential equations governing mass transfer in an electrolytic system into Laplace's equation for the quasi-potential and a set of coupled first-order, nonlinear ordinary differential equations. The first part, namely Laplace's equation, is referred to as the geometry-specific part since it accounts for the geometry and is independent of the specific chemical (or electrochemical) system used. The latter part is independent of the geometry and is referred to as the system-specific part. The advantage in using this transformation is that the resulting set of ordinary differential equations is relatively easy to solve. Furthermore, there is a vast body of literature detailing solutions to Laplace's equation subject to electrochemical-type boundary conditions. Conversely, the original set of governing equations can be difficult or impossible to solve without the introduction of restrictive conditions such as the neglect of migration as a mechanism of mass transfer. Another advantage is that the geometry-specific and system-specific parts are independent and can thus be readily combined to make the solutions for many geometries and systems easily accessible.

Certain conditions apply to the use of the quasi-potential transformation. The convective contribution to the mass transfer must be negligible. We have not been able to solve systems in which multiple heterogeneous reactions take place. In the problems we have 
solved, it has been found necessary that all homogeneous reactions be equilibrated. The transformation applies only to steady-state or quasi-steady-state systems.

We have expanded the work of Baker, Verbrugge, and Newman [1], by developing a theoretical framework for the quasi-potential transformation. Our basis is the assumption that the concentrations can be written as single-valued functions of the electrostatic potential (or the concentration of an arbitrarily chosen species). From this assumption we have shown that the stagnant species satisfy Boltzmann distributions in the solution, using a theorem given by Gibbs [8]. Baker, Verbrugge, and Newman start with the assumption that the stagnant species satisfy Boltzmann distributions in the solution, and use this to show that the concentrations can be written as single-valued functions of the electrostatic potential. While both approaches give the same result, the basis we have used is the more general in nature.

These authors have also restricted the use of the transformation to systems in which only one ion participates in the electrode reaction. We have discussed how the transformation may be used for an electrode reaction in which multiple ions participate. The examples they have treated are for arbitrary ionic species with no homogeneous reactions taking place, and the transport properties are considered constant. We have modeled the binary and acidic coppex sulfate systems. For the binary system we have studied the effect of considering the transport properties to be variable and for the acidic system 
we have considered the incomplete dissociation of bisulfate ions. In so doing we have shown how the quasi-potential transformation may be used to treat homogeneous reactions and have examined the effect of considering the dissociation constant to be constant with respect to local ionic strength. We have also developed a geometry-independent expression that allows us to quantify the effect of migration on limiting currents in systems to which the quasi-potential transformation is applicable. 
References

[1] D. R. Baker, M. W. Verbrugge, and J. Newman, "A transformation for the treatment of diffusion and migration. Application to stationary disk and hemisphere electrodes," J. Electroanal. Chem., $314,23-44$ (1991).

[2] B. Levich, "The Theory of Concentration Polarization," Acta Physicochimica U.R.S.S, 17(5-6), 257-307 (1942).

[3] R. M. Wightman and D. O. Wipf, "Voltammetry at Ultramicroelectrodec," in Electroanalytical Chemistry, 15, edited by A. J. Bard, Marcel Dekker Inc., New York (1987).

[4] S. M. Sharland, "A Review of the Theoretical Modelling of Crevice and Pitting Corrosion," Corrosion Science, 27(3), 289-323 (1987).

[5] J. Newman, Electrochemical Systems, Prentice Hall, Englewood Cliffs, New Jersey (1991).

[6] H. S. Carslaw and J. C. Jaeger, Conduction of Heat in Solids, Oxford University Press, New York (1959).

[7] G. K. Batchelor, An Introduction to Fluid Dynamics, Cambridge University Press, Cambridge (1967).

[8] J. W. Gibbs, The Scientific Papers Vol. II, Dover Publications Inc., New York (1961). 
[9] A. C. West and J. Newman, "Determining Current Distributions Governed by Laplace's Equation," in Modern Aspects of Electrochemistry, submitted.

[10] J. Newman, "The Fundamental Processes of Current Distribution and Mass Transport in Electrochemical Cells," in Electroanalytical Chemistry, 6, edited by A. J. Bard, Marcel Dekker Inc., New York (1973).

[11] J. Newman, "Resistance for Fiow of Current to a Disk," J. Electrochem. Soc., 113(5), 501-502 (1966).

[12] L. Nanis and W. Kesselman, "Engineering Applications of Current and Potential Distributions in Disk Electrode Systems," J. Electrochem. Soc., 118(3), 454-461 (1971).

[13] C. R. Wylie and L. C. Barrett, Advanced Engineering Mathematics, McGraw-Hill Publishing Company, New York (1982).

[14] M. Abramowitz and I. A. Stegun, Handbook of Mathematical Functions, Dover Publications Inc., New York (1964).

[15] E. Mattsson and J. O'M. Bockris, "Galvanostatic Studies of the Kinetics of Deposition and Dissolution in the Copper + Copper Sulphate System," Trans. Faraday Soc., 55, 1586-1601 (1959).

[16] U. Bertocci, "Application of Electrochemical Theory to the Behaviour of Copper in Cupric and Cuprous Solutions, "Electrochimica Acta, 11, 1261-1277 (1966). 
[17] O. R. Brown and H. R. Thirsk, "The Rate-Determining Step in the Electrodeposition of Copper on Copper from Aqueous Cupric Sulphate Solutions," Electrochimica Acta, 10, 383-393 (1965).

[18] D. G. Miller, J. Rard, L. B. Eppstein, and R. A. Robinson, "Mutual Diffusion Coefficients, Electrical Conductances, Osmotic Coefficients, and Ionic Transport Coefficients $1_{i j}$ for Aqueous $\mathrm{CuSO}_{4}$ at $25^{\circ} \mathrm{C}, " \mathrm{~J}$. Solution Chem., $9(7), 467-496$ (1980).

[19] M. J. Pikal and D. G. Miller, "Hittorf Transference Numbers in Aqueous Copper Sulfate at $25^{\circ} \mathrm{C}, " \mathrm{~J}$. Chem. Eng. Data, 16(2), 226 229 (1971).

[20] T. F. Young, L. F. Maranville, and H. M. Smith, "Raman Spectral Investigations of Ionic Equilibria in Solutions of Strong Electrolytes," Chapter 4 in The Structure of Electrolytic Solutions, edited by W. J. Hamer, John Wiley and Sons Inc., New York (1959).

[21] L. Hsueh and J. Newman, "The Role of Bisulfate Ions in Ionic Migration Effects," Ind. and Eng. Chem. Fundamentals, 10, 615$620(1971)$.

[22] H. M. Goodwin and W. G. Horsch, "The Electrical Conductivity and Other Properties of Saturated Solutions of Copper Sulphate in the Presence of Sulphuric Acid," Chem. and Metallurgical Eng., $21(4), 181-182$ (1919). 
Appendix A

Computer Programs

The programming language used here is SVS Pascal (Silicon Valley Software). The source code shown here uses only two constructs that are not part of the 1980 ISO standard. The variable type double is a double-precision real-type variable. The rewrite command, that enables the CPU to identify the data file, is a nonstandard construct. The programs were compiled and run on a DUAL Systems Corporation system $83 / 80$, which has a Motorola 68451 CPU chip.

The programs listed below are those programs that were used to obtain the values displayed in the various figures in this work. In order to avoid unnecessary duplication, all the variations on these programs have been omitted.

PROGRAM CULM

The program will return the values of the quantities of interest in the binary $\mathrm{CuSO}_{4}$ solution, at constant potential distribution on the electrode, using the fourth-order Runge-Kutta algorithm. In the form shown, this program was used to obtain values for the limitingcurrent case. It was altered slightly to generate values of the quasi-potential adjacent to the surface as a function of the bulk concentration of $\mathrm{CuSO}_{4}$ (Figure 2).

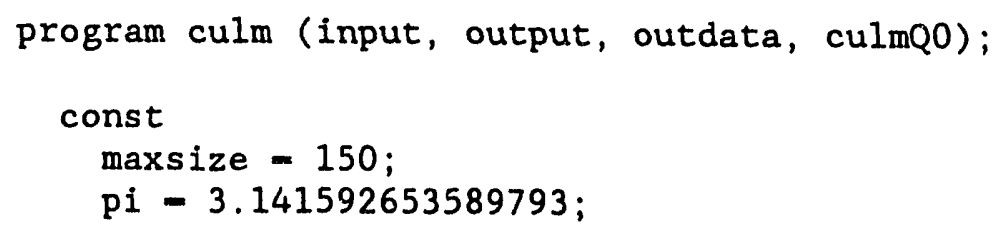




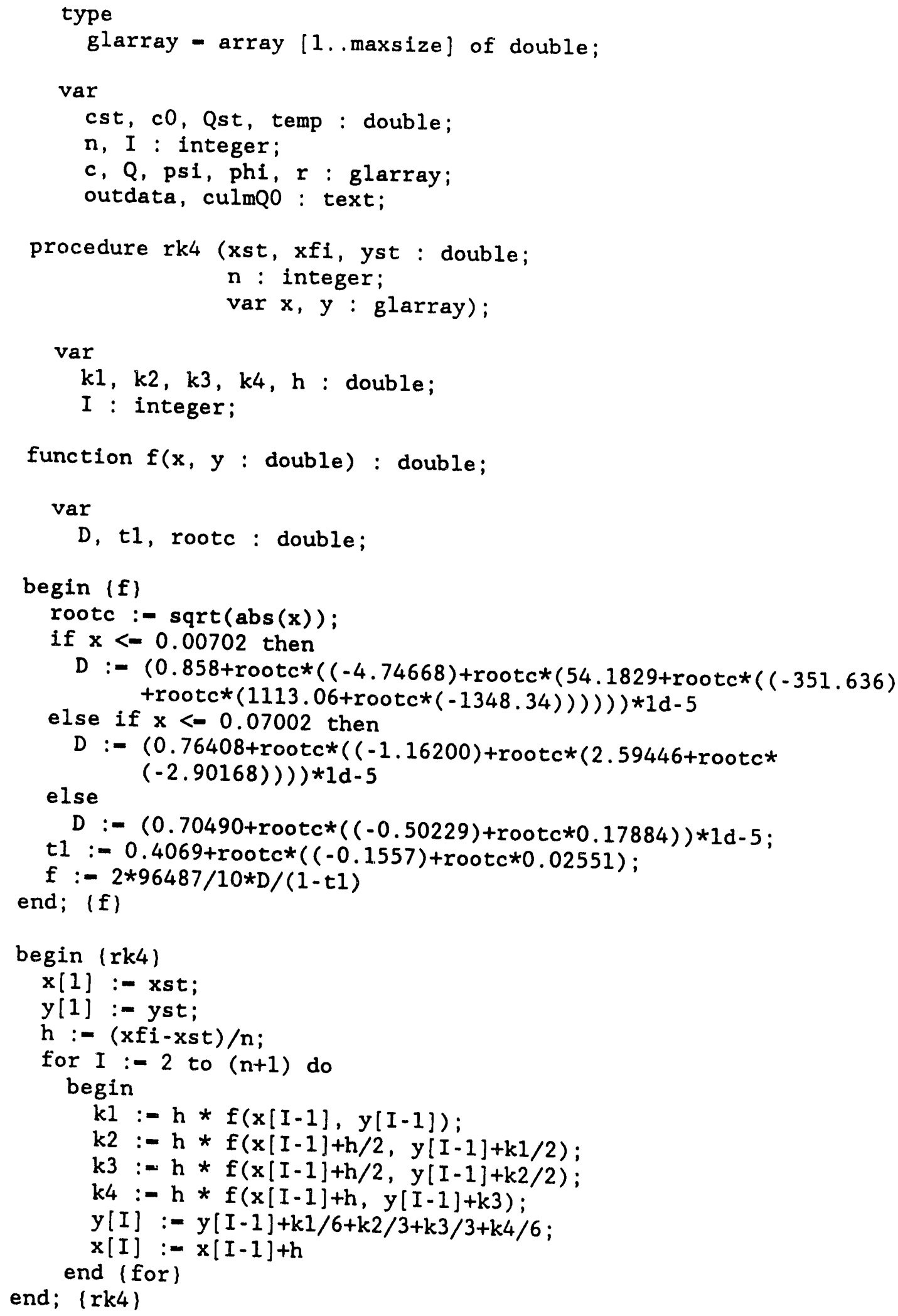




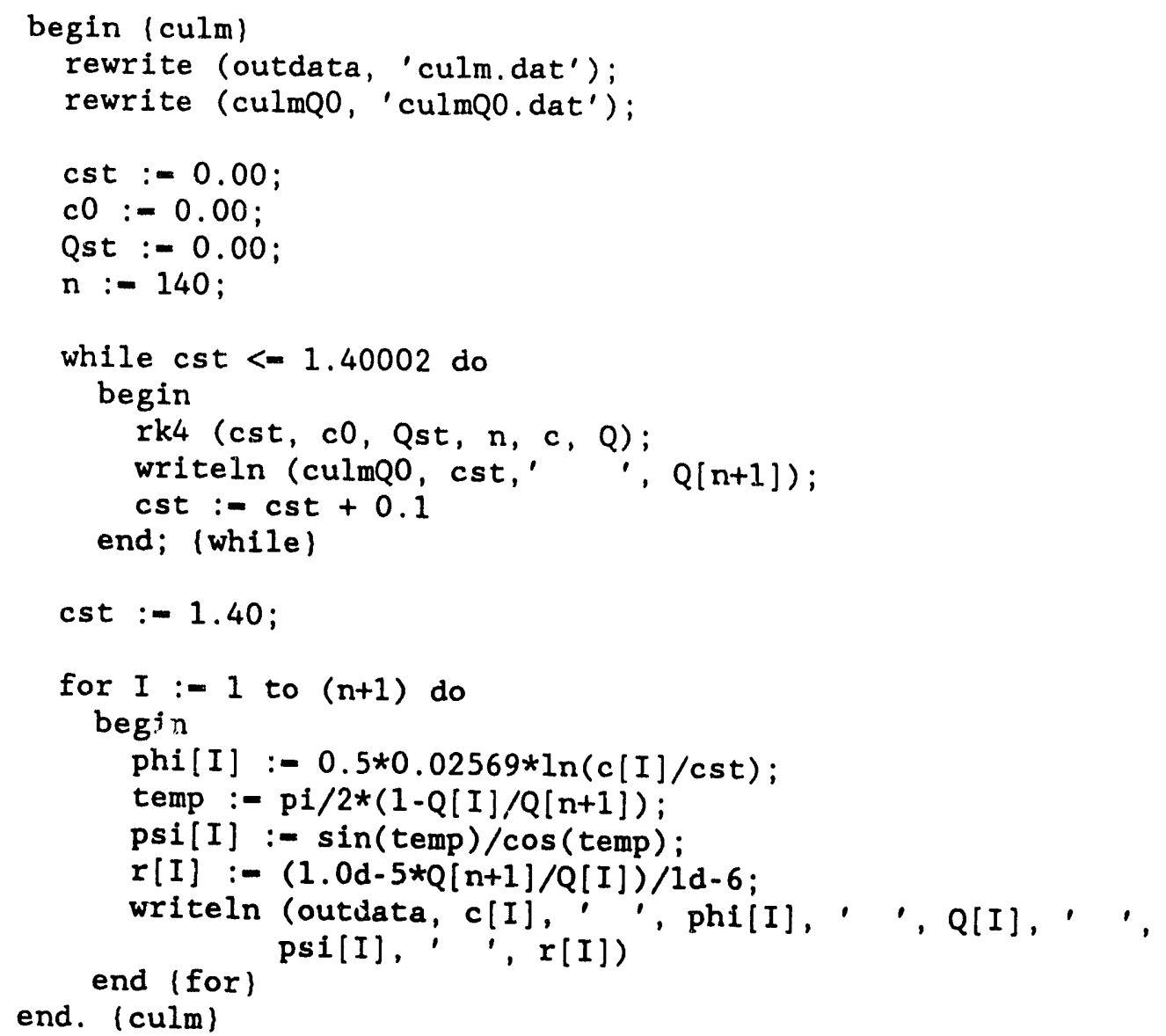

\section{PROGRAM ACID}

This program uses the fourth-order Runge-Kutta algorithm to solve for the quantities of interest in the acidic $\mathrm{CuSO}_{4}$ solution, at constant potential distribution. As shown it evaluates these properties at the limiting-current distribution. As with program culm, this program had to be slightly modified to obtain the values of the quasi-potential on the surface as a function of bulk concentration of $\mathrm{CuSO}_{4}$, as shown in figure 9.

program acid (input, output, outdata); 


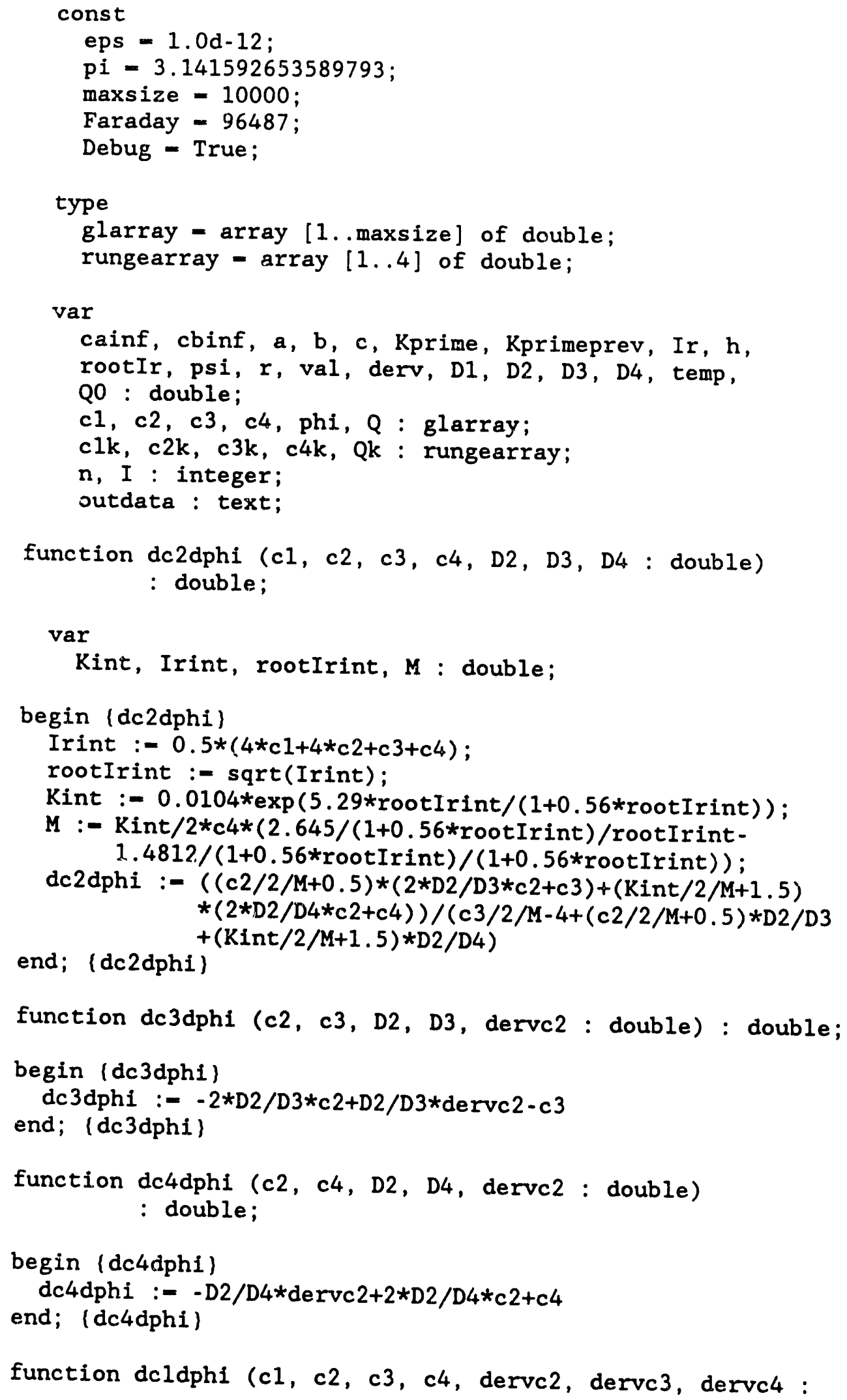




$$
\text { double) : double; }
$$

var

Kint, Irint, rootIrint, $M$ : double;

begin (dcldphi)

Irint : $=0.5 *(4 * c 1+4 * c 2+c 3+c 4)$;

rootIrint :- sqrt(Irint);

Kint : $=0.0104 * \exp (5.29 *$ root Irint $/(1+0.56 *$ root Irint $))$;

$M:-$ Kint $/ 2 * c 4 *(2.645 /(1+0.56 *$ rootIrint $) /$ rootIrint $1.4812 /(1+0.56 *$ rootIrint $) /(1+0.56 *$ rootIrint $))$;

dc1dphi :- dervc $2 *(c 3 / 4 / M-1)+\operatorname{dervc} 3 *(c 2 / 4 / M-0.25)$

end; \{dcldphi\} -dervc4*(Kint $/ 4 / M+0.25)$

function dQdphi (c1, c2, c3, c4, dervc1, dervc2, dervc3, dervc4, D1, D2, D3, D4 : double) : double;

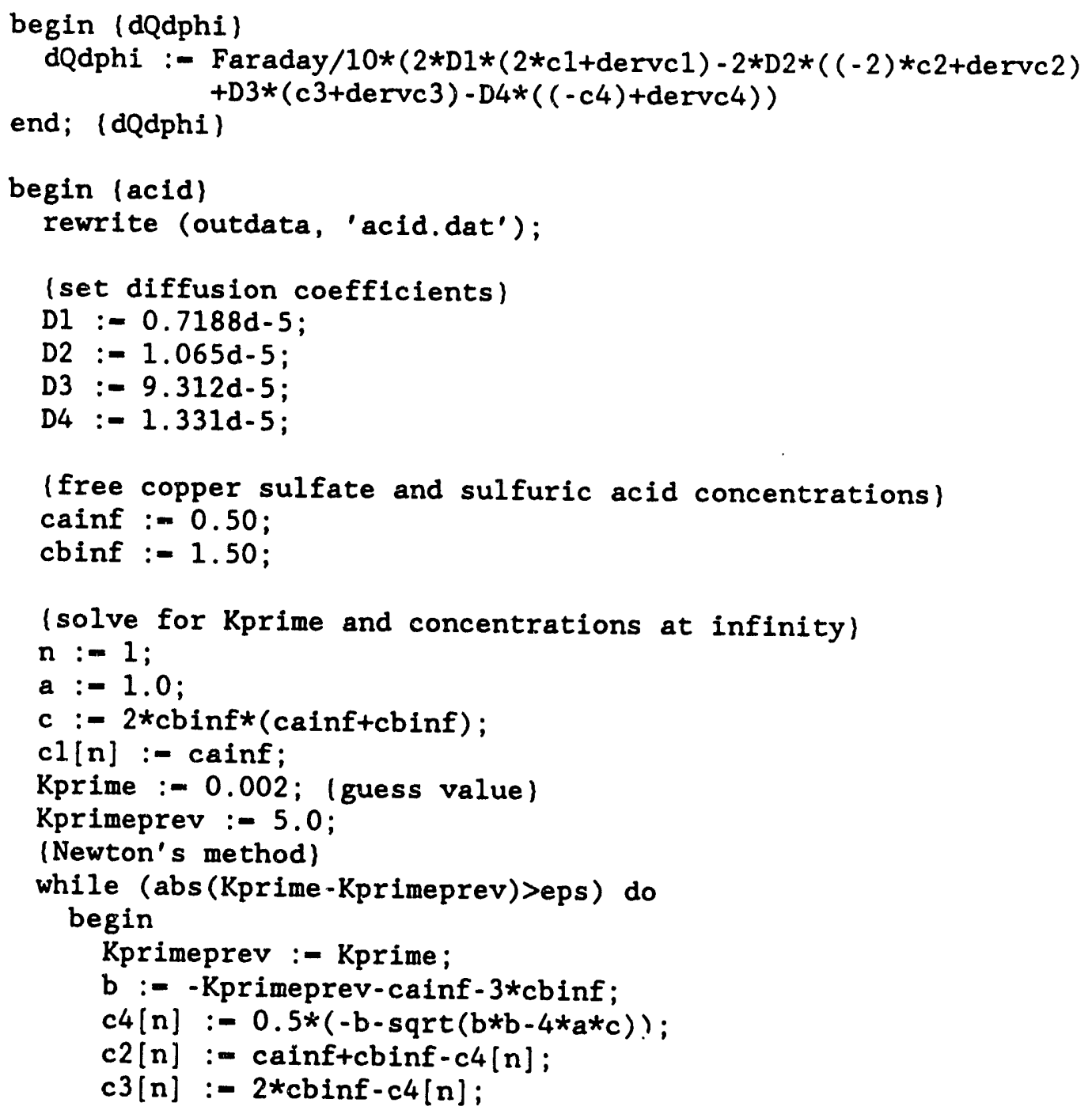




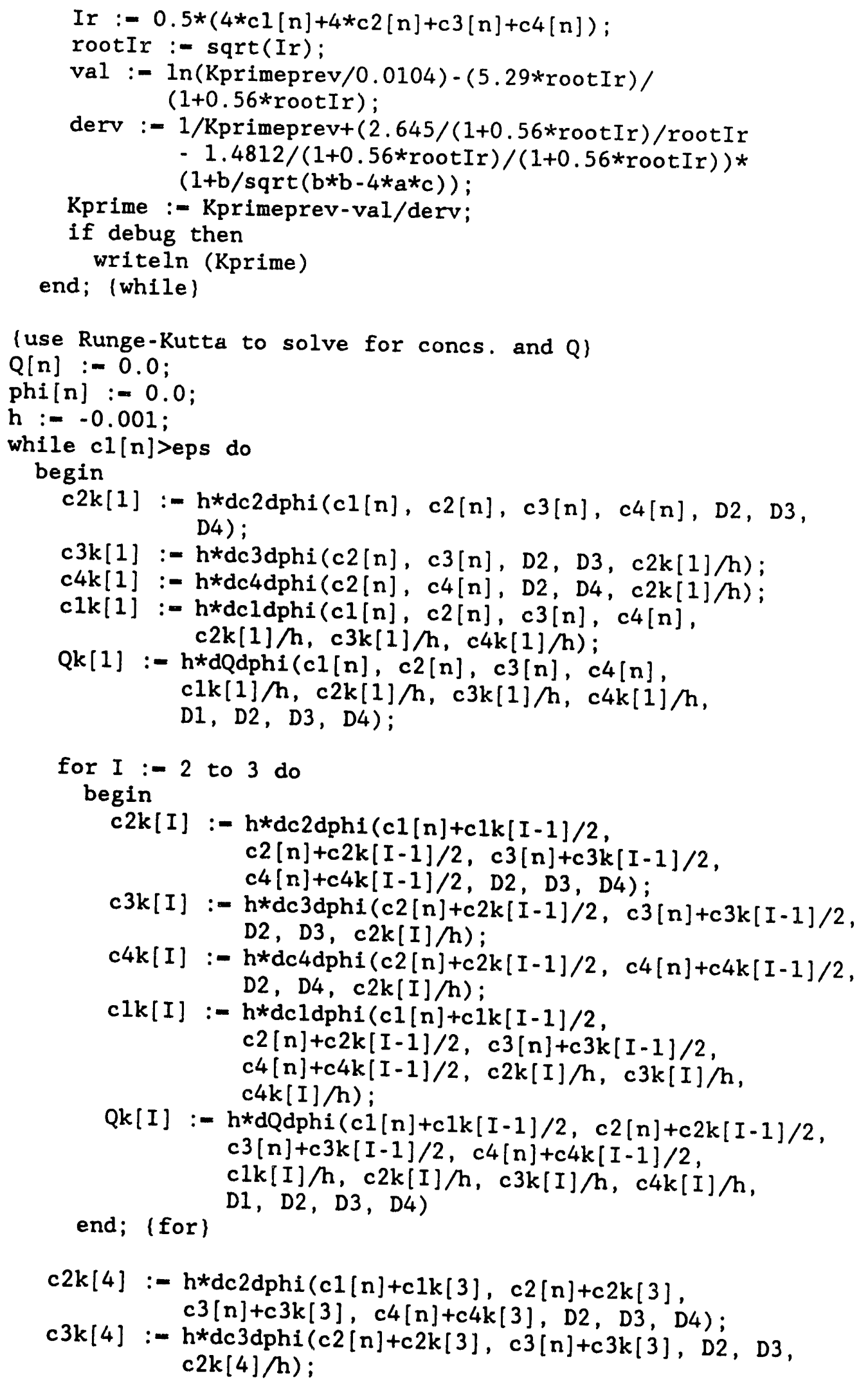




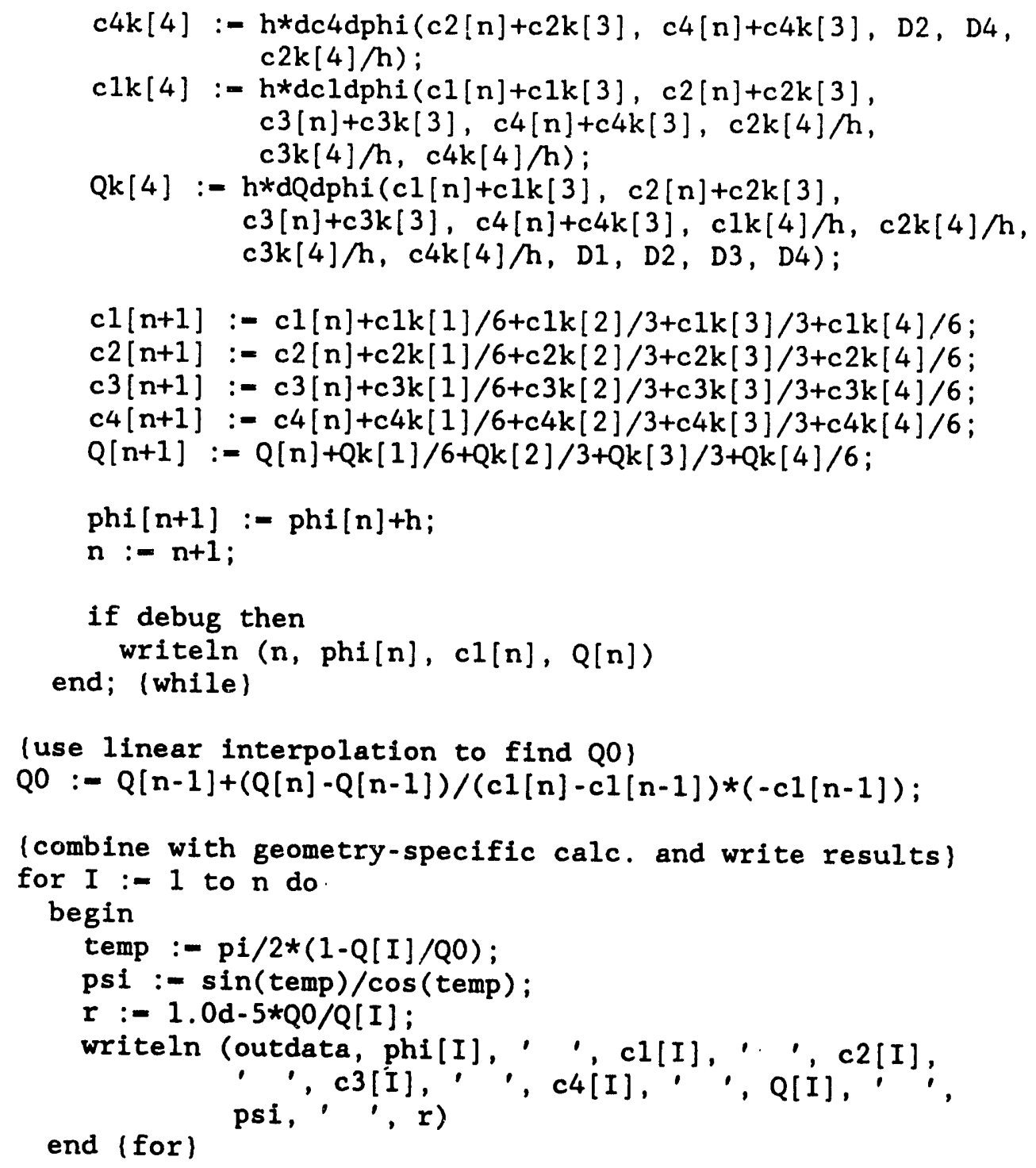

end. (acid)

PROGRAM CUUCDDSK

This program uses Newton's method and an implementation of the fourth-order Runge-Kutta algorithm, that is almost identical to that used in the program culm, to obtain the variation of electrostatic potential adjacent to a disk electrode, in binary $\mathrm{CuSO}_{4}$ solution, as shown in figure 7. A variation of this program, using the 
implementation of the fourth-order Runge-Kutta algorithm from the program acid, is used to obtain the analogous result for acidic $\mathrm{CuSO}_{4}$ solution, as shown in figure 12 .

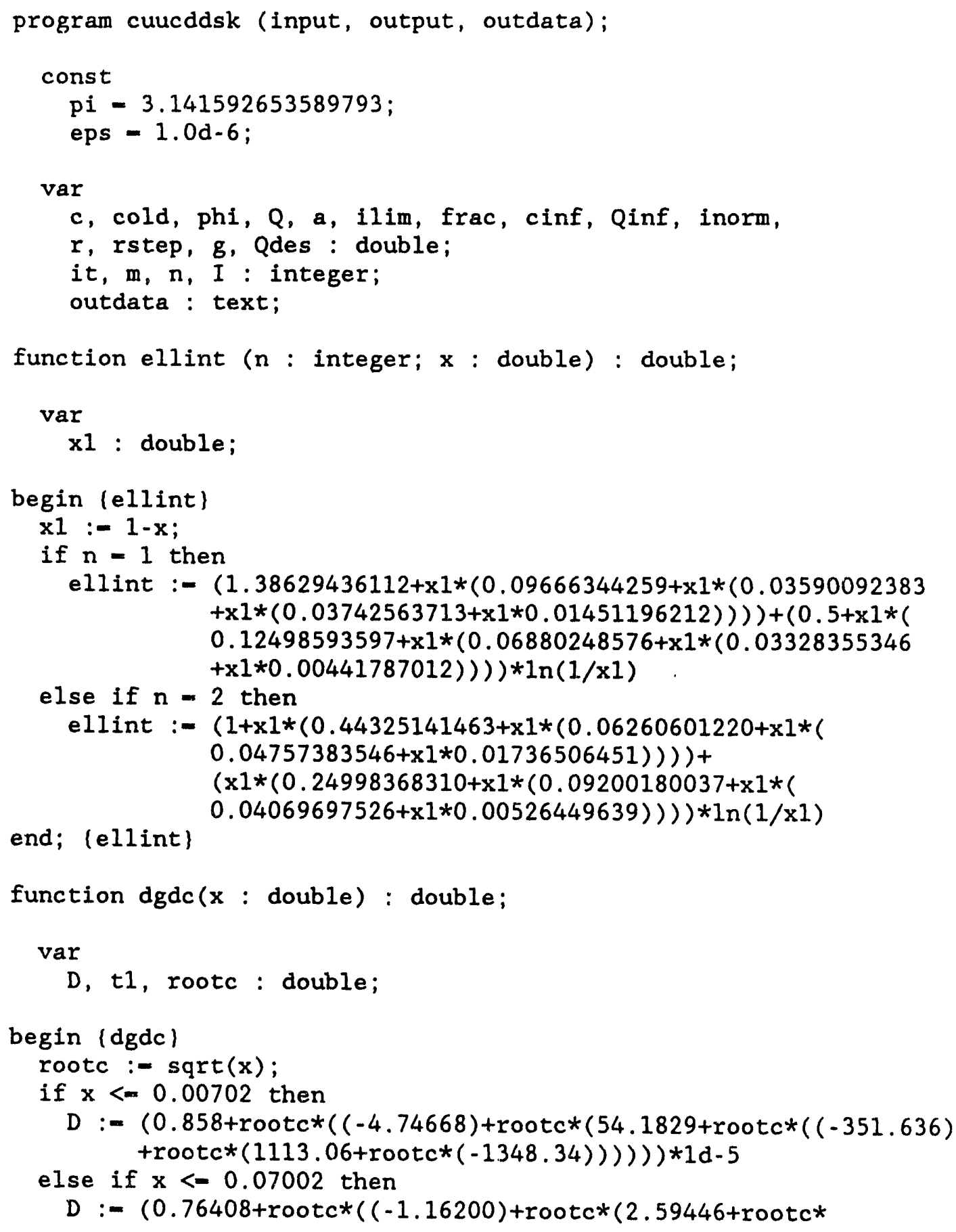




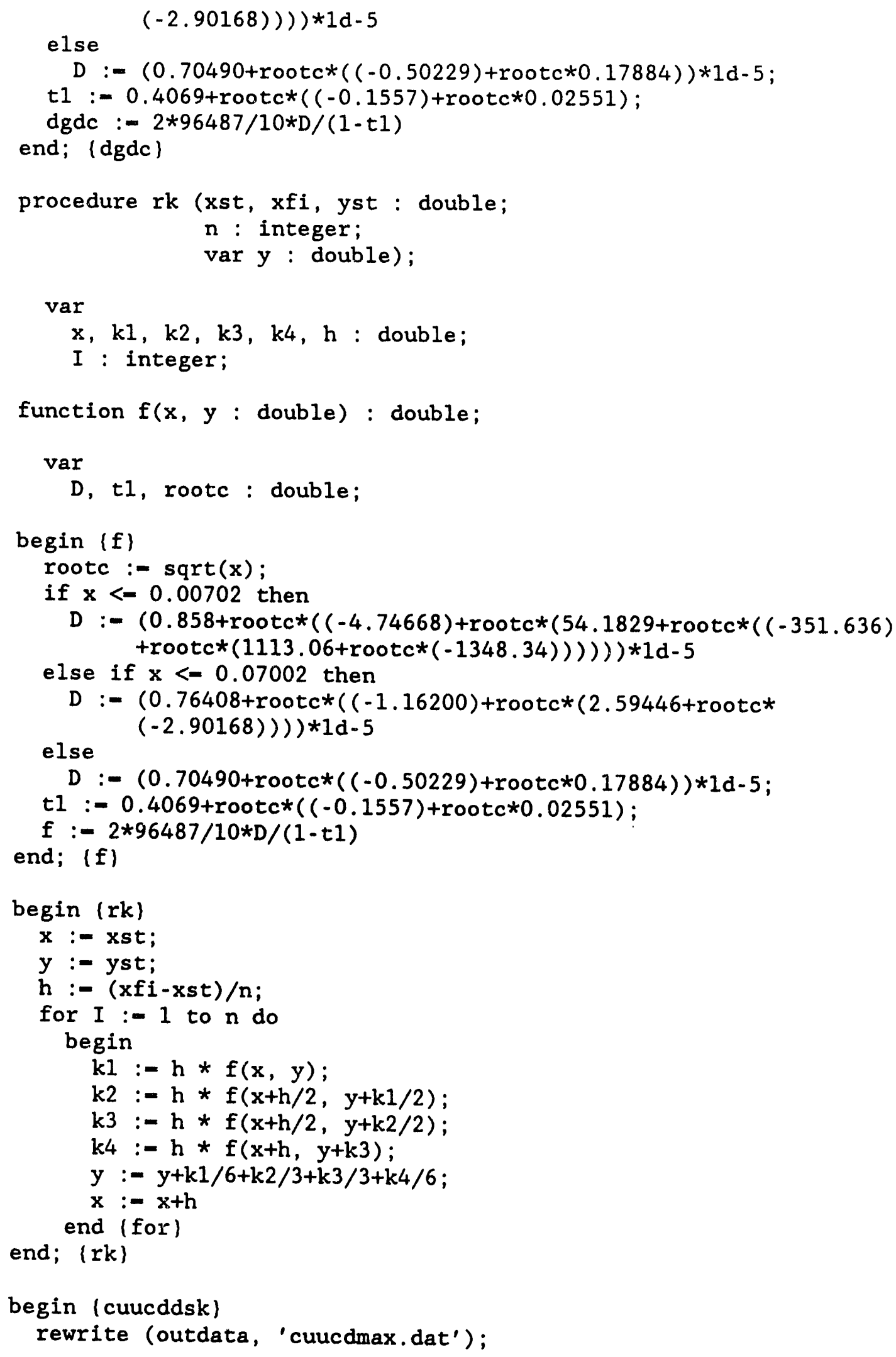




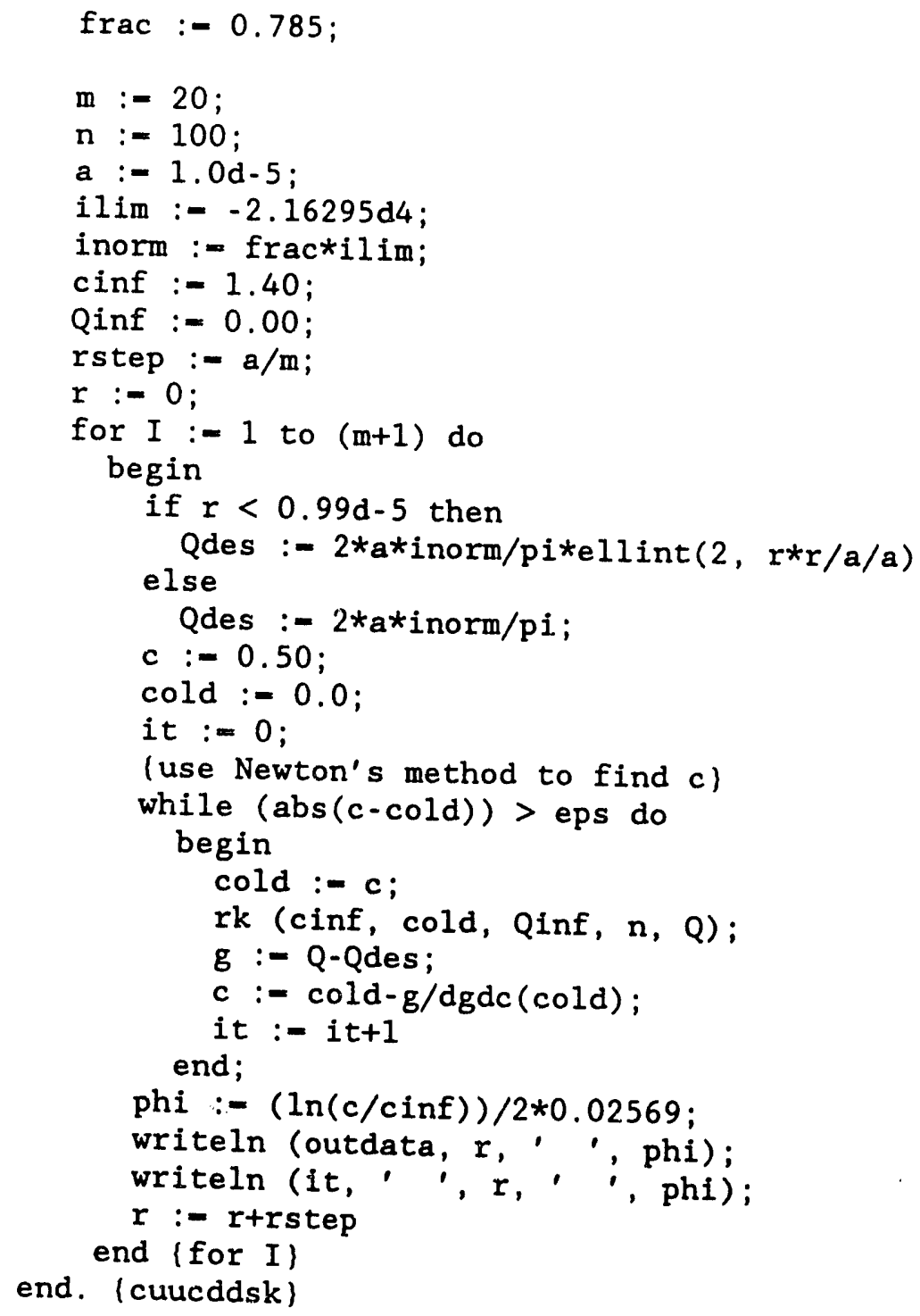



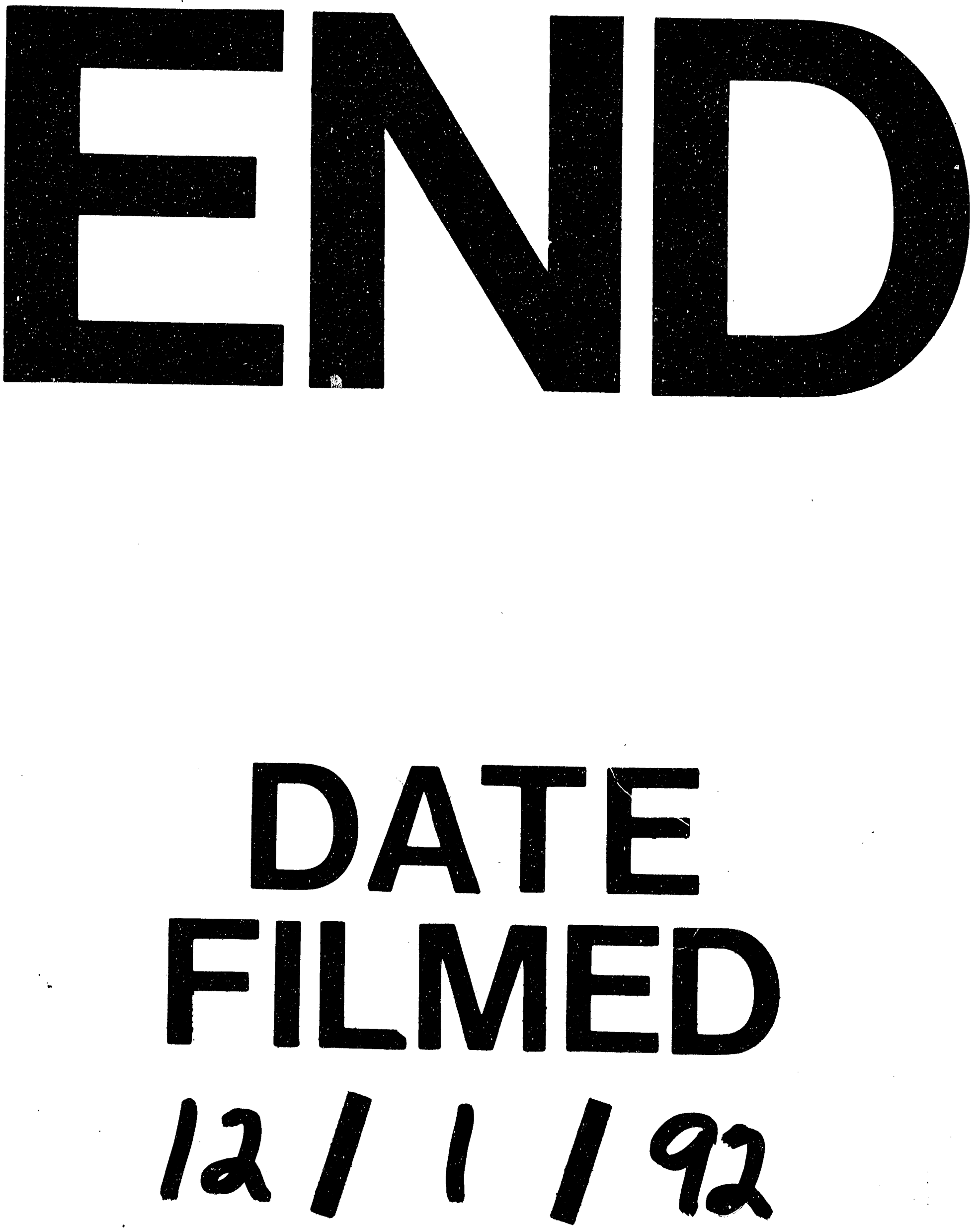
